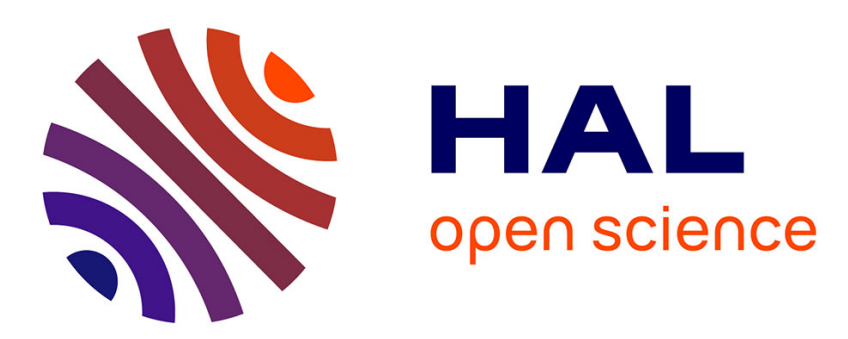

\title{
On gravity currents of fixed volume that encounter a down-slope or up-slope bottom
}

\author{
Tamar Zemach, Marius Ungarish, Antoine Martin, Maria-Eletta Negretti
}

\section{To cite this version:}

Tamar Zemach, Marius Ungarish, Antoine Martin, Maria-Eletta Negretti. On gravity currents of fixed volume that encounter a down-slope or up-slope bottom. Physics of Fluids, 2019, 31 (9), pp.096604. 10.1063/1.5121290 . hal-02391195

\section{HAL Id: hal-02391195 \\ https://hal.science/hal-02391195}

Submitted on 15 Jan 2020

HAL is a multi-disciplinary open access archive for the deposit and dissemination of scientific research documents, whether they are published or not. The documents may come from teaching and research institutions in France or abroad, or from public or private research centers.
L'archive ouverte pluridisciplinaire HAL, est destinée au dépôt et à la diffusion de documents scientifiques de niveau recherche, publiés ou non, émanant des établissements d'enseignement et de recherche français ou étrangers, des laboratoires publics ou privés. 


\title{
On gravity currents of fixed volume that encounter a down-slope or up-slope bottom
}

\author{
T. Zemach, ${ }^{1, a)}$ M. Ungarish, ${ }^{2, b)}$ A. Martin, ${ }^{3}$ (D) and M. E. Negretti ${ }^{3}$
}

\author{
AFFILIATIONS \\ 1 Department of Computer Science, Tel-Hai College, Tel-Hai, Israel \\ ${ }^{2}$ Department of Computer Science, Technion, Haifa, Israel \\ ${ }^{3}$ LEGI, UGA/CNRS UMR 5519 Grenoble Cedex 9, France \\ a)tamar.zemach@yahoo.com \\ b)unga@cs.technion.ac.il
}

\begin{abstract}
We consider a gravity current released from a lock into an ambient flui of smaller density, that, from the beginning or after some horizontal propagation $X_{1}$, propagates along an inclined (up- or down-) bottom. The flo (assumed in the inertial-buoyancy regime) is modeled by the shallow-water (SW) equations with a jump condition applied at the nose (front). The behavior of the current is dominated by the slope angle, $\theta$, but is also affected by additional dimensionless parameters: the aspect ratio of the lock $x_{0} / h_{0}$, the height ratio of the ambient to lock, $H / h_{0}$, and the distance of the backwall from the beginning of the slope, $X_{1} / x_{0}$. We show that the stability of the interface, reflecte by the value of the bulk Richardson number, $R i$, is essential in the interpretation and modeling. In the upslope flow $R i$ increases and hence entrainment/mixing effects are unimportant. In the downslope flow the current firs accelerates and $R i$ decreases; this enhances entrainment and drag, which then decelerate the current. We show that the accelerating-decelerating downstream current is reproduced well by a SW model combined with a simple closure for the entrainment and drag. A comparison of the theoretical results with previously published experimental data for both upslope flo and downslope flo show fair agreement.
\end{abstract}

\section{INTRODUCTION}

Gravity currents (GCs) are ubiquitous in many geophysical and environmental flow such as salt intrusions into lakes and estuaries, glacial runoff into the ocean, turbidity currents in coastal regions, cold downhill airflow in mountain areas, or snow avalanches. In oceans, dense currents descend the continental slope for long distances before encountering the ocean bottom or interleaving at their level of neutral buoyancy (e.g., Ref. 1). In mountain areas, the dynamics of the atmospheric boundary layer is dominated by downslope currents. In enclosed mountain valleys, pollutants emitted by traffic heating/cooling systems, and industry transported by katabatic winds are mainly trapped at low altitudes (Ref. 11) with well-known important consequences on human health. Avalanches are natural hazards that are observed quite frequently in winter and spring and which cause relevant damages to our infrastructures such as buildings, roads, and electric power transmission and can cost life to humans. While the generating mechanism of avalanches remains quite unpredictable, their development is strongly dependent on the characteristics of the terrain as the slope angle variations (e.g., Ref. 15).

The typical gravity current of one density $\left(\rho_{c}\right)$ is released from a lock of length $x_{0}$ and height $h_{0}$ into another flui of a different density $\left(\rho_{a}\right)$ and is driven by the reduced gravity $g^{\prime}=\left(\rho_{c} / \rho_{a}-1\right) g$, where $g$ is the gravitational acceleration and we assume $\rho_{c}>\rho_{a}$. Let $x_{N}(t)$ denote the horizontal distance of propagation as a function of time and $u_{N}(t)$ denote the corresponding speed of propagation of the nose (front).

In many cases of interest, the gravity current (GC) encounters a topography, i.e., an upslope or downslope. ${ }^{2,5,10,16,28}$ These problems have received recent attention in the works of Refs. 22, 8, and 21. These studies emphasize the experimental observation and are therefore focused on a rather restricted range of parameters. Indeed, the dimensions of typical laboratory tanks pose severe limitations on the size of the lock, length of the inclined slope, and angle $\theta$. For a comprehensive understanding and prediction of the flow 
a general model, built on clear-cut equations of motion, accepting realistic initial and boundary conditions, and not reliant on adjustable constants, is necessary. Unfortunately, such a model has not been presented and tested, to the best of our knowledge.

The experiments (e.g., Refs. 22 and 8) reveal that in downslope flow the current firs accelerates and then decelerates. These observations are routinely compared with the model (referred to as "power law" or "thermal theory") of Ref. $3 x_{N}=\xi_{0}+K\left(g^{\prime} h_{0} x_{0}\right)^{1 / 3}(t+$ $\left.\tau_{0}\right)^{2 / 3}$ (dimensional), where $\xi_{0}, \tau_{0}$ are adjustable constants, and the dimensionless $K$ is given by a formula which contains the entrainment and drag coefficient $E, C_{d}$ and some adjustable shape-factors. Thermal theory describes both the acceleration phase and the equilibrium state of the current; herein, the common approach is based on a balance between the momentum and mass conservation equations of the finit volume released gravity current. This assessment is based on the following limiting assumptions: (1) geometric simplification of the shape of the finit volume released flo (e.g., halfellipse, rectangle), (2) self-similarity, and (3) empirical estimates of entrainment and drag coefficients

For the upslope current, the authors of Ref. 21 developed an approximation that predicts constant deceleration and hence provided a simple algebraic estimate to the position where the current stops $\left(u_{N}=0\right)$, under the assumption that the speed at the base of the slope is known. This, again, employs some bold assumptions (e.g., the nose Froude number is a constant). It is worthy to emphasize the differences between the ingredients of the down-slope and up-slope propagation formulas of Refs. 3 and 21: the firs uses entrainment and drag but ignores the front $\mathrm{Fr}$ condition; the second uses the front $\mathrm{Fr}$ condition but discards entrainment and drag. This is intriguing because essentially this is the same gravity current, released from the same lock. Here, we attempt to close this gap of knowledge.

Our starting point is the horizontal current. There is evidence that for a high-Reynolds-number gravity current, the shallow-water
(SW) theory with a Fr number jump condition at $x_{N}$ predicts well the dam-break process after release from the lock (see Ref. 30). The SW equations can be applied to nonhorizontal boundaries. However, it turns out that the interpretation of the flo in the presence of $|\theta|>0$ and the elucidation of the differences between the up- and down-slope cases are not straightforward. To this end, solutions of the SW equations (by finit difference method) are needed, and, at a later stage, the reason for significan entrainment and the extension of the SW model must be addressed. Support to this unifie theoretical approach is sought in comparisons with available experimental data. This is the objective of our paper.

We note that SW models for an inclined bottom have been used by Ref. 20. However, that work was focused on a special configura tion of full-depth lock in a tank with an open top, incorporated from the beginning adjustable parameters, and lacked a clear-cut $F r$ jump condition. This precludes the insights and versatility attainable by the present simpler SW model.

The paper is organized as follows. The shallow-water formulation is presented in Sec. II, and the finite-differenc method used for the solution is presented in Sec. III. Theoretical predictions for down-slope and up-slope cases are discussed in Secs. III A and III B. Comparisons with experiments, presented in Sec. IV, show good agreement for the upslope case but less satisfactory performance for the downslope case. The reasons are discussed in Sec. IV B 1, and it is shown that the remedy for the downslope configuratio is an extended SW model which includes the effects of entrainment and drag. Concluding remarks are given in Sec. V.

\section{FORMULATION}

Consider a gravity current created by release of a fixe volume constant density $\rho_{c}$ and kinematic viscosity $v$ into an ambient flui of constant density $\rho_{a}$. The system is sketched in Fig. 1 . The current is propagating in the $x$-direction in a channel with a partially inclined

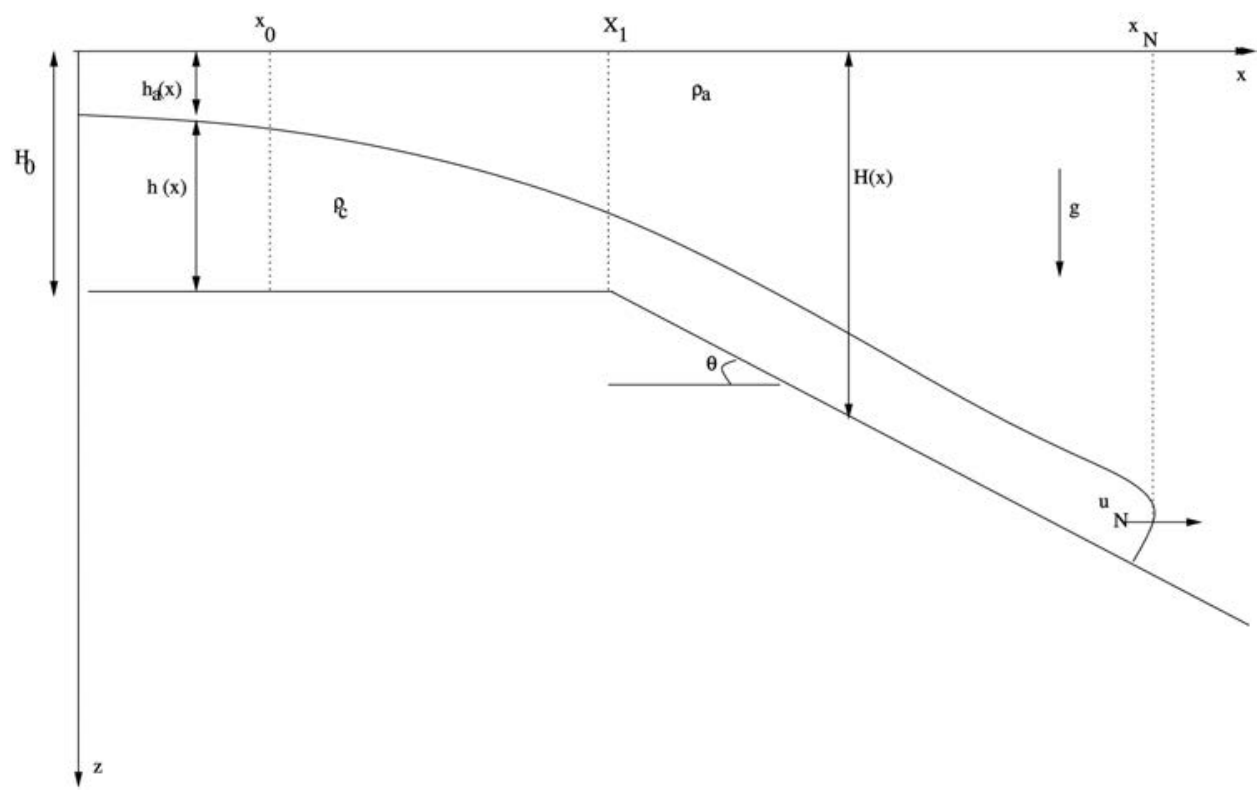

FIG. 1. Schematic description of the current released from a lock of length $x_{0}$ and height $h_{0}$ in a channel of height $H(x)$ with a horizontal top and a bottom slope starting at $x=X_{1} \cdot \tan \theta=-d H / d x$. 
bottom. The height of the channel $H(x)$ is dependent on $x$ and is assumed to be constant for $x \leq X_{1}$ and to be a linear function of $x$ for $x>X_{1}$.

The system under consideration is sketched in Fig. 1: the top and the bottom of the half-infinit channel are at $z=0$ and $z=H(x)$. Gravity acts in the $z$ direction. At time $t=0$, a given volume of flui of density $\rho_{c}>\rho_{a}$, initially at rest in reservoir of height $h_{0}$ and length $x_{0}$, is instantaneously released into the ambient fluid A twodimensional $\{x, z\}$ Cartesian coordinate system with corresponding $\{u, w\}$ velocity components is employed. The fluid are assumed to be separated by a sharp, nonentraining interface (this will be reconsidered later). Let the subscripts $c$ and $a$ denote the current and the ambient domains. The height of the current is $h(x, t)$, and that of the ambient flui is $h_{a}(x, t)=H(x)-h(x, t)$. The SW approximation assumes that the current is thin (formally, $h_{0} / x_{0} \ll 1$ ), and hence the motion can be represented by the $z$-averaged (over the thickness of the layer) velocity of the current $u(x, t)$ and of the ambient flui $u_{a}(x, t)$. The task is to obtain the equations of motion. The methodology has been discussed in the literature (e.g., Refs. 30 and 31). Here, we briefl show some details which are of importance to the nonhorizontal bottom configuration

Let $p$ denote the pressure. The SW approximation implies that in the vertical direction, the velocity and acceleration are small, and therefore, the pressure (a) obeys the hydrostatic balance and (b) is continuous at the interface $z=h(x, t)$. This can be expressed as

$$
\begin{aligned}
& p_{a}(x, z, t)=\Phi(x, t) \\
& p_{c}(x, z, t)=\Delta \rho g\left(z-h_{a}\right)+\Phi(x, t),
\end{aligned}
$$

where $\Delta \rho=\rho_{c}-\rho_{a}$.

The pressure gradient in the current does not dependent on $z$, and it can be written as

$$
\frac{\partial p_{c}}{\partial x}=-\Delta \rho g \frac{\partial h_{a}}{\partial x}+\frac{\partial \Phi(x, t)}{\partial x}
$$

The Reynolds number of the flow $R e=h_{N} u_{N} / v$, where the subscript $N$ denotes value associated with the "nose" of the current, is assumed large. Since the viscous terms are negligible, the $x$-pressure gradient is balanced by the inertial terms. The resulting set of continuity equations and inertial-pressure ( $x$-momentum balance) of the problem are

$$
\left\{\begin{array}{l}
\frac{\partial h}{\partial t}+\frac{\partial}{\partial x}(u h)=0, \\
\frac{\partial h_{a}}{\partial t}+\frac{\partial}{\partial x}\left(u_{a} h_{a}\right)=0 \\
\frac{\partial u}{\partial t}+\frac{\partial}{\partial x}\left(\frac{1}{2} u^{2}\right)=-\frac{1}{\rho_{c}}\left[-\Delta \rho g \frac{\partial h_{a}}{\partial x}+\frac{\partial \Phi}{\partial x}\right] \\
\frac{\partial u_{a}}{\partial t}+\frac{\partial}{\partial x}\left(\frac{1}{2} u_{a}^{2}\right)=-\frac{1}{\rho_{a}} \frac{\partial \Phi}{\partial x} .
\end{array}\right.
$$

The firs two equations in system (2.3) represent conservation of volume in each layer, and the next two equations represent balance of horizontal momentum in a hydrostatic pressure fiel in each layer.

A useful reduction of system (2.3) is obtained by the elimination of the ambient-layer variables. The upper boundary is considered a fixe top, and there is no flo through the $x=0$ backwall. This yields

$$
h+h_{a}=H(x)
$$

and

$$
u h+u_{a} h_{a}=0 .
$$

Combining (2.3) with (2.4) and (2.5) provides, after some algebra, the set of governing equations for the variables $h(x, t)$ and $u(x, t)$ of the current,

$$
\left\{\begin{array}{l}
\frac{\partial h}{\partial t}+\frac{\partial}{\partial x}(u h)=0 \\
\frac{\partial u}{\partial t}\left[1+\frac{\rho_{a}}{\rho_{c}} \frac{a}{1-a}\right]+u \frac{\partial u}{\partial x}\left[1-\frac{\rho_{a}}{\rho_{c}} \frac{a(1+a)}{(1-a)^{2}}\right]+\frac{\partial h}{\partial x}\left[\frac{\Delta \rho}{\rho_{c}} g-\frac{\rho_{a}}{\rho_{c}} u^{2} \frac{1}{H(1-a)^{3}}\right]=\frac{d H}{d x}\left[\frac{\Delta \rho}{\rho_{c}} g-\frac{\rho_{a}}{\rho_{c}} u^{2} \frac{a^{2}}{H(1-a)^{3}}\right]
\end{array}\right.
$$

Here, $a(x)=\frac{h}{H(x)}$. Recall that $\tan \theta \sim-d H / d x$. For the noninclined bottom, $H=$ const., $\theta=0$, the last term of the second equation of (2.6) vanishes, and system (2.6) reduces to the standard two-layer non-Boussinesq system of equations [see Ref. 31, Eqs. (2.1) and (2.3)]. On the other hand, the inclination contributes a significan forcing term $\propto \tan \theta$ in the momentum equation.

It is convenient to use dimensionless variables define as follows (here, the dimensional variables are denoted by an asterisk):

$$
\left\{x^{*}, z^{*}, h^{*}, H^{*}, t^{*}, u^{*}\right\}=\left\{x_{0} x, h_{0} z, h_{0} h, h_{0} H, T t, U u\right\},
$$

where

$$
U=\sqrt{\frac{\Delta \rho}{\rho_{c}} g h_{0}} ; T=\frac{x_{0}}{U}
$$

In the characteristic dimensionless form, the equations become

$$
\left(\begin{array}{l}
h \\
u
\end{array}\right)_{t}+\left(\begin{array}{cc}
u & h \\
B & (1-2 A) u
\end{array}\right)\left(\begin{array}{l}
h \\
u
\end{array}\right)_{x}=\left(\begin{array}{c}
0 \\
C \cdot \frac{d H}{d x}
\end{array}\right) .
$$

Here,

$$
\begin{aligned}
& A=\frac{1}{1-a+R a} \frac{R a}{1-a}, \\
& B=\frac{1}{1-a+R a}\left[1-a-\frac{R}{H(1-a)^{2}} u^{2}\right], \\
& C=\frac{1}{1-a+R a}\left[1-a-\frac{R}{H(1-a)^{2}} u^{2} a^{2}\right],
\end{aligned}
$$


and $R$ is define by

$$
R=\frac{\rho_{a}}{\rho_{c}}
$$

The Boussinesq system corresponds to $R \approx 1$. In the formulation, we simply substitute $R=1$.

System (2.9) is hyperbolic. The relationships between the variables on the characteristics are as follows:

$$
\begin{aligned}
d u & =\frac{u(1-2 A)-\lambda_{ \pm}}{h} d h+C \frac{d H}{d x} d t \text { on } \\
\frac{d x}{d t} & =\lambda_{ \pm}=u(1-A) \pm \sqrt{A^{2} u^{2}+h B} .
\end{aligned}
$$

The left-hand side of (2.9) does not include $H$ explicitly, and it is identical to this obtained for the horizontal, noninclined surface. However, it depends on $a$, which is a function of $H(x)$. As a result, the eigenvalues and the eigenvectors are also identical to those obtained for the noninclined surface but again for $a=a(x)$. An additional difference between the systems of equations is the presence of the source term in the momentum equation of the inclined surface. We note that when $\frac{d H}{d x}=0$, Eq. (2.9) become a standard formulation of the noninclined two-layer system. Another simple case is for $\frac{d H}{d x}=\frac{x_{0}}{h_{0}} \tan \theta$. This case will be discussed in detail later.

\section{A. Initial and boundary conditions}

The initial and boundary conditions are $u=0, h=1$ at $t=0$ in the lock $0 \leq x \leq 1$ and $u=0$ at the backwall $x=0$. To obtain realistic gravity current solutions, the system must be subjected to a boundary condition at the nose $x=x_{N}(t)$. The justificatio is provided by analogy to the horizontal noninclined dam-break problem. ${ }^{19,30}$ Indeed, the dam-break activates the characteristics of the hyperbolic system. The forward-moving characteristics intersect and form a jump. The control-volume analysis about this thin jump is dominated by the volume flu and flow-forc on the vertical planes and is not affected by the inclination of the bottom (assuming a nonlarge $|\tan \theta|)$. Consequently, the instantaneous jump condition for the nose is like in the horizontal case. ${ }^{4}$ In dimensionless form, this reads

$$
u_{N}=\frac{1}{R^{1 / 2}} \operatorname{Fr}\left(a_{N}\right) h_{N}^{1 / 2},
$$

where $a_{N}=h_{N} / H\left(x_{N}\right)$ and $\operatorname{Fr}\left(a_{N}\right)$ is the Froude number function, define by

$$
\operatorname{Fr}\left(a_{N}\right)=\sqrt{\frac{(2-a)(1-a)}{1+a}} .
$$

\section{B. Critical nose region}

The flui cannot travel faster than the perturbation (characteristic) that sustains the motion, i.e., $u_{N} \leq \lambda_{+}$. The critical behavior occurs when $u_{N}=u_{c r i t}=\lambda_{+}$, and in general, the propagation is subcritical with $h_{N} \leq h_{\text {crit }}$ and $u_{N} \leq u_{\text {crit }}$. The critical conditions are obtained by the analysis as used for $2 \mathrm{~L}$ non-Boussinesq currents propagating in noninclined containers [see Ref. 31, Eq. (3.19)]. The analysis is valid here, in particular, because as we already mentioned above, the eigenvalues and eigenvectors for the inclined or partially inclined problems are formally identical to those obtained for the noninclined container for $a=a(x)$. The result is

$$
h_{\text {crit }}=0.3473 H\left(x_{N}\right) ; u_{\text {crit }}=0.5273 \frac{\sqrt{H\left(x_{N}\right)}}{\sqrt{R}} .
$$

The difference with the horizontal case is the fact that $H(x)$ varies. This implies that $u_{\text {crit }}$ increases in a down-slope motion. In this configuration the critical conditions are important because the current tends to concentrate behind the nose.

\section{Critical angles of validity and viscosity effects}

The critical angles for the validity of the model depend on the transition of the dynamics from upslope current to an impact/splash (e.g., Refs. 13 and 27) for gravity currents flowin upslope. For the downslope currents, it has been shown by Beghin et al. ${ }^{3}$ that the front speed continuously varies with the slope angle (ranged between $5^{\circ}$ and $90^{\circ}$ ). However, Baines ${ }^{1}$ showed that for a continuously supplied gravity flow a transition from gravity current to turbulent plume depends on the slope angle and the buoyancy forces. By definition, the shallow water solution as presented here relies on the hydrostatic balance assumption which fails when the slope angle of the current becomes large: for instance, when $\theta=30^{\circ}$, the vertical velocity becomes of the same order as the mean stream velocity (factor $1 / 2$ ). To our knowledge, no corresponding systematic study is available in the literature determining clearly a range of validity of the shallow water approach for increasing slope angles.

For this study, a large Reynolds number is assumed for the shallow water model; the prediction therefore neglects viscosity which exerts a nontrivial effect as the front approaches its maximum height for the upslope current. Marleau et al. ${ }^{21}$ reported that viscous effects can be important in the last stage of the propagation of the upslope current on the slope when the current becomes thin. However, drag coefficien is also highly related to the Reynolds number. For a downslope flow the bottom friction is often neglected for a high Reynolds number. In a viscous boundary layer, $C_{d}$ is inversely proportional to Reynolds numbers as is also shown by Cenedese and Whitehead. In Cenedese and Whitehead, the drag coefficien $C_{d}$ increases with the inverse of $R e$ define from the peak velocity of the gravity current. For a wall jet (that has a velocity profil similar to a gravity current) of Reynolds number larger than $1000, C_{d}$ is smaller than $5 \times 10^{-3} \cdot{ }^{12}$ For a turbulent boundary layer, $C_{d}$ is given by the empirical relation $C_{d} \approx 0.0113, R^{-0,178} \approx 3 \times 10^{-3}$, obtained from the classical relation $C_{d}=0.027 / R e_{x}^{1 / 7}$ with $x=\delta R e_{x}^{1 / 5} / 0.37$, which is close to the bottom drag coefficien for a wall jet at the same Reynolds number.

\section{FINITE-DIFFERENCE RESULTS}

The solution of the equations must be obtained numerically. Since for the classical noninclined containers, efficien solutions were obtained with a two-step Lax-Wendroff method (see Ref. 6), we attempted an extension which incorporates the contributions of the slope of the container. Here, we use a two-step Lax-Wendroff finite difference method to calculate by time-marching from given initial conditions the height $h(x, t)$, the velocity $u(x, t)$, and the distance of propagation $x_{N}(t)$ of the current from the system of equations [(2.9) and (2.13)]. 
The present work is limited to a linear-inclined bottom for which in dimensionless form, $H(x)$ can be represented by

$$
H(x)= \begin{cases}H_{0}, & 0 \leq x \leq X_{1}, \\ H_{0}+\left(x-X_{1}\right) \cdot \tan \theta \cdot \frac{x_{0}}{h_{0}}, & X_{1}<x \leq x_{N} .\end{cases}
$$

And therefore,

$$
\frac{d H}{d x}= \begin{cases}0, & 0 \leq x \leq X_{1}, \\ \tan \theta \cdot \frac{x_{0}}{h_{0}}, & X_{1}<x \leq x_{N} .\end{cases}
$$

The free input parameters of the problem are (1) the density ratio $R=\frac{\rho_{a}}{\rho_{c}}$, (2) the height of the container nonslope part $H_{0}$, (3) the length $X_{1}$ where the slope starts, (4) the sloping angle $\theta$, and (5) the ratio between the initial height and length $x_{0} / h_{0}$. We note that $X_{1}$ is a non-negative value. The case $X_{1}=0$ reproduces a fully inclined bottom; the case $X_{1}=\infty$ describes the standard noninclined configuration The present model covers two different configurations down slope with $\theta>0$ and increasing function $H(x)$ (for $x>X_{1}$ ) and up slope with $\theta<0$ and decreasing function $H(x)$ (for $x>X_{1}$ ). We wish to emphasize that the aspect ratio of the lock, $x_{0} / h_{0}$, enters the scaled balances for the inclined current. To be more precise, the scaled influenc of the slope is $\propto\left(x_{0} / h_{0}\right) \tan \theta$. The interpretation is geometric. When the nose propagates to $x_{0}$, the vertical displacement is $\Delta z=x_{0} \tan \theta$. The ratio $\Delta z / h_{0}$ is an estimate of the change of pressure driving force excess relatively to the horizontal motion.

The SW results displayed here were obtained with, typically, 200 grid points in the $\left[0, x_{N}\right]$ interval and time step of $1 \times 10^{-3}$. (Convergence was tested also on fine grids.) We note that the numerical computation requires insignifican computer time and memory on a laptop.

For simplicity of analysis and interpretation, the results presented here are for Boussinesq systems, $R=1$.

\section{A. Downslope case, $\theta>0$}

The system configuratio for this case is shown in Fig. 1.

\section{The effect of the slope location $X_{1}$}

Some results obtained for the $\theta=10^{\circ} ; R=1 ; H_{0}=1.2 ; x_{0} / h_{0}$ $=1$ and various locations of the slope start position $X_{1}=0,0.5,1$, 2 are shown in Figs. 2-5. We analyze the behavior of the current during the short times $(t \leq 2.5)$ until the back-moving perturbation approaches the left wall. We observe some wiggles in the numerical solution, which is a spurious by-product of the truncation errors (numerical diffusion and dispersion, see the work of Morton and Mayers $^{24}$ ) and has a negligible influenc on the accuracy of the solution at other points.

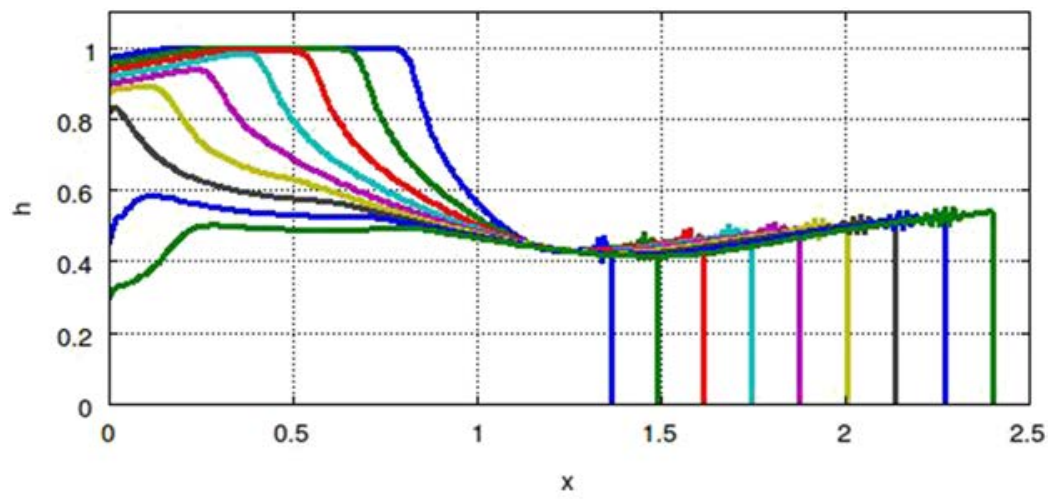

(a)

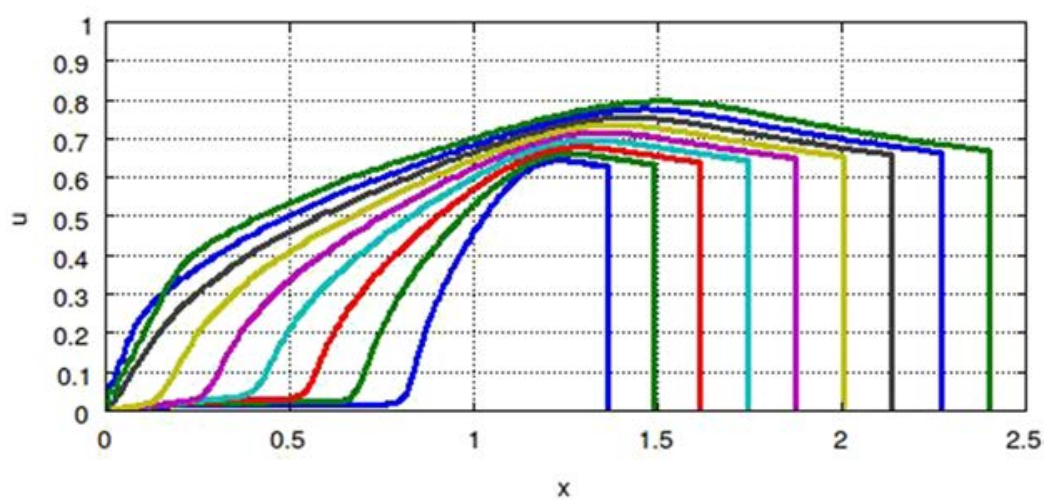

(b)
FIG. 2. SW model results for the down-slope bottom case: (a) $h$ and (b) $u$ as functions of $x$ at various $t=0.4(0.2) 2.0$. Here, $\theta=10^{\circ} ; X_{1}=0 ; H_{0}=1.2 ; x_{0} / h_{0}=1$. 


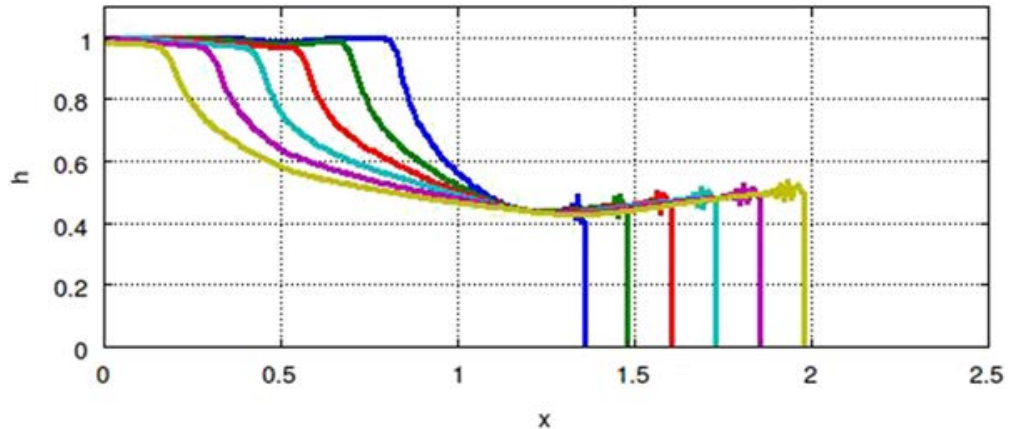

(a)

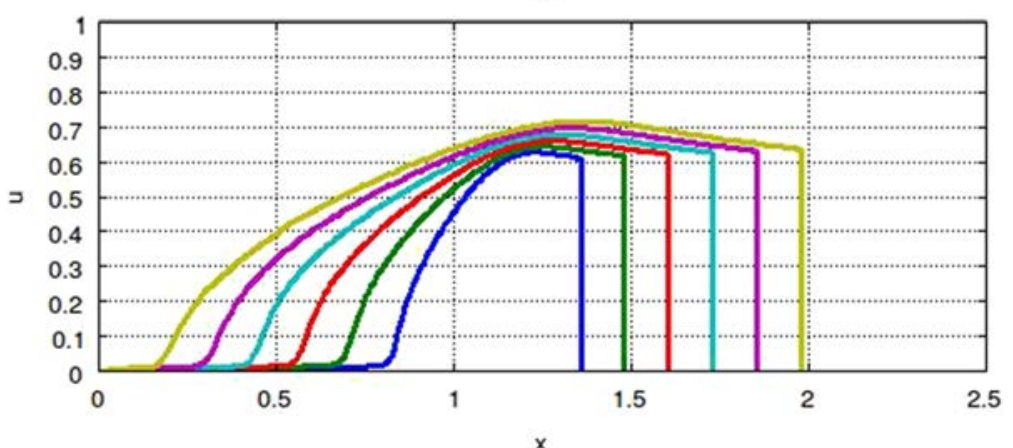

(b)

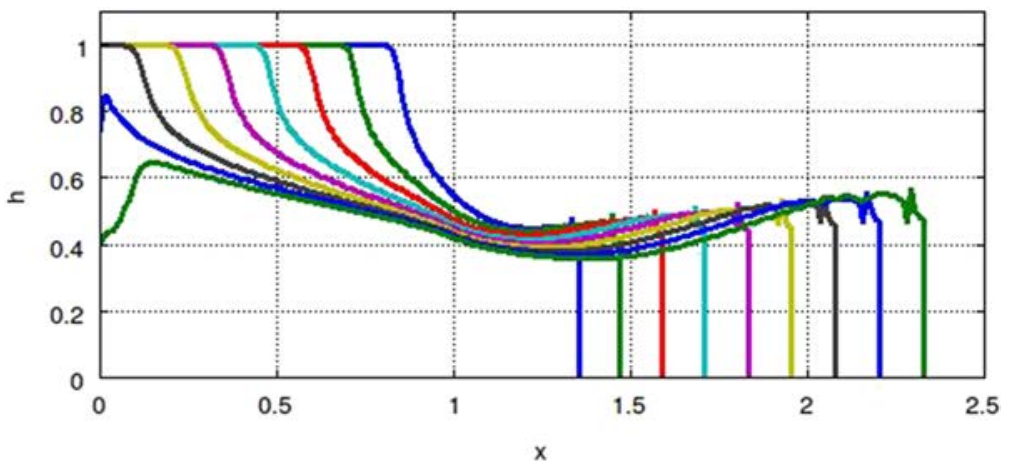

(a)

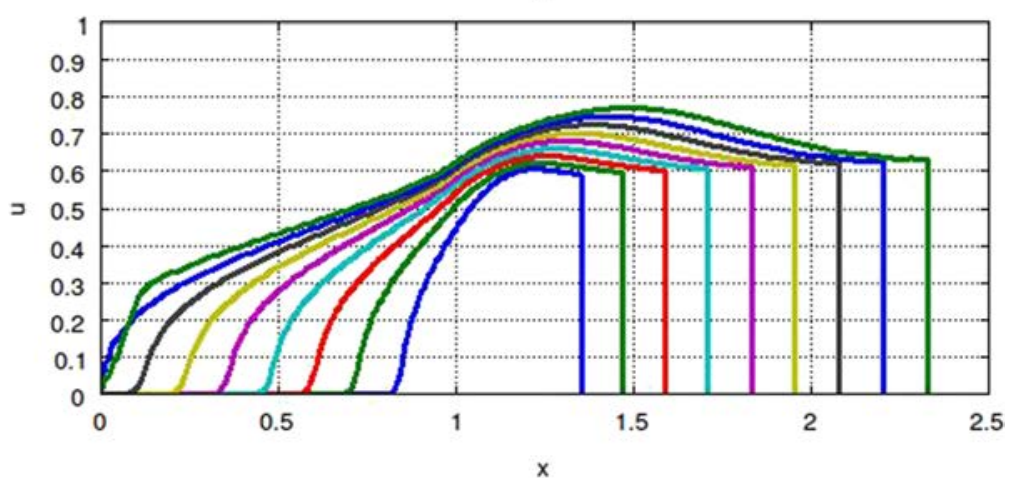

(b)
FIG. 3. $X_{1}=0.5 . t=0.4(0.2) 1.4$. Other parameters are as in Fig. 2.
FIG. 4. $X_{1}=1.0$. Other parameters are as in Fig. 2. 


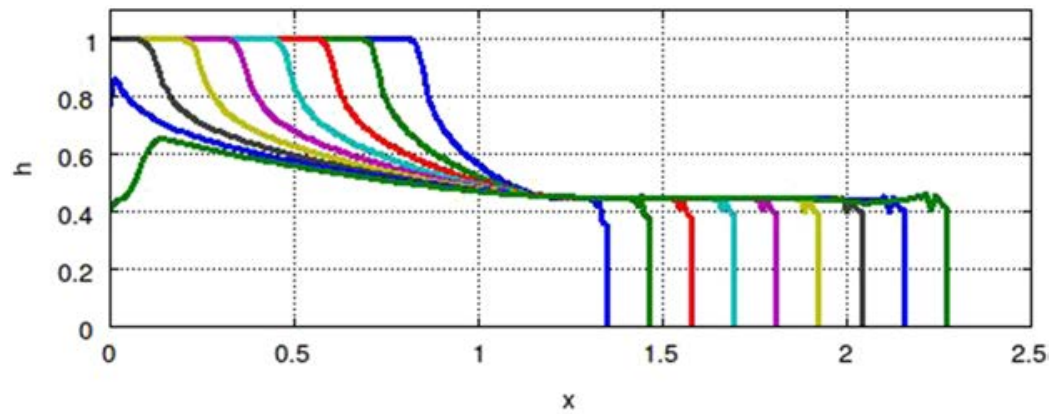

(a)

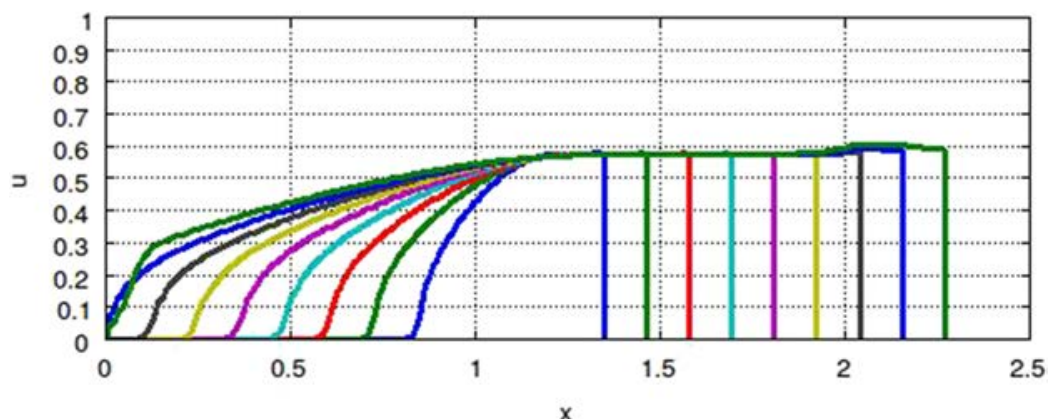

(b)
FIG. 5. $X_{1}=2.0$. Other parameters are as in Fig. 2.
As expected, as $X_{1}$ increases, the inclined bottom starts "later," so the current propagates slower. We note that for early times, the behavior of the currents in containers with $X_{1}=2$ and $X_{1}=3$ and $\theta=10^{\circ}$ is identical to this obtained for the horizontal noninclined system with $\theta=0^{\circ}$. We speculate that the reason of such propagation is that during early stages, the current still "does not know" that the bottom is inclined because the back-moving front approaches the left wall before the current nose approaches $X_{1}$. For $X_{1}<2$, however, the situation is different and the current is slightly faster than the one propagating in the horizontal case. Moreover, as expected, as $X_{1}$ decreases (until 0 ), the speed of propagation of the current increases. Figure 6 shows the distance of propagation of the current for various values of $X_{1}$. Other parameters are as in Fig. 5.

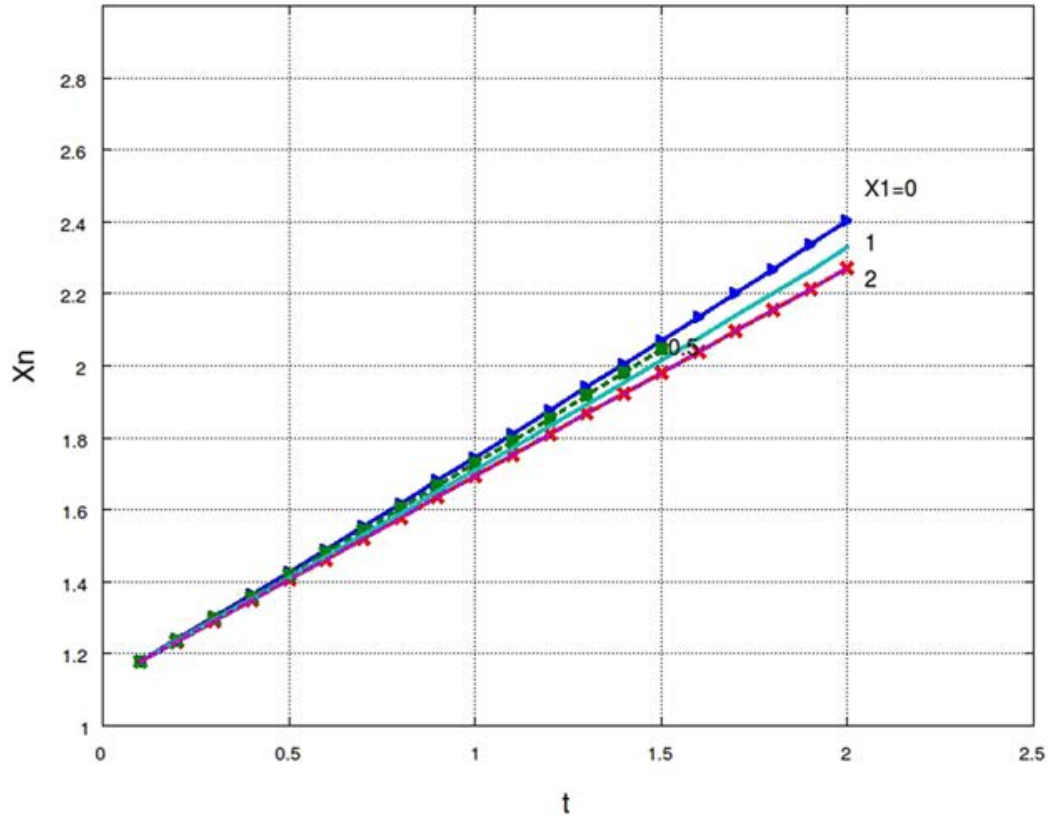

FIG. 6. Distance of propagation of the current for various locations of the sloping start positions $X_{1}=0,0.5,1,2$ in the downslope case. Other parameters are as in Fig. 2. The lines for the noninclined container and for $X_{1}=2$ coincide. 
An additional interesting effect is the behavior of the height $h$ at around $x \approx X_{1}$. Usually, in the noninclined containers, at the beginning of the motion, the height of the current $h$ is constant and equals to 1 in some area $0 \leq x \leq x_{c}(t)$. This area decreases with time and disappears when the left-moving perturbation approaches the left wall. However, in inclined or bottom containers, the height of the current might not be constant already during the firs stages of propagation. The perturbation starts at the location which is very close to the beginning of the slope $X_{1}$ and is spreading to the left direction.

The firs stage of propagation of the current moving in the noninclined bottom case is the so-called "slumping" stage during which the height $h_{N}$ and the speed $u_{N}$ of the current nose are constant (for a significan period of time).

The behavior of the currents in the inclined bottom case, however, is different and depends on the position of the slope. Thus, for $X_{1}<2$, the speed of the nose $u_{N}$ increases from the beginning until the left-moving perturbation approaches the left wall by a moderate value of about $5 \%$. The nose height $h_{N}$ also increases but much more dramatically by a rate of about $30 \%$ for $X_{1}=0$. The rate of increasing becomes more moderate as the position of slope, $X_{1}$, moving right and finall in containers with bottom-slope starting at $X_{1} \geq 2, h_{N}$, remains constant.

\section{The effect of the slope angle $\theta$}

Results obtained for $\theta=15^{\circ}$ (as before, $H_{0}=1.2, R=1, X_{1}=1$ ) are shown in Fig. 7. In general, as expected, the current propagates faster than the corresponding $\theta=10^{\circ}$ case since the increase in the slope accelerates the velocity of propagation. However, quantitatively, while $\theta$ increases by $50 \%$ (from $10^{\circ}$ to $15^{\circ}$ ), the height $h_{N}$ and the speed $u_{N}$ of the nose increase by few percents only.

\section{The effect of the container height $H(x)$ and spreading at the large times}

Above we discussed the propagation of the current in containers with the height ratio $H_{0}$ close to 1 . Here, we discuss the behavior of the currents in a deep-height container with $H_{0}=3$. In particular, our interest is the form of the currents obtained at the progressive times and their difference from those obtained in Sec. III A 1 and III A 2. Figure 8 shows the behavior of the current in the container with $\theta=10^{\circ} ; X_{1}=1 ; H_{0}=3$ during its propagation to progressive times $t=0.5-10.5$. The initial stage of spreading is similar to that shown for $H=1.2$ in Fig. 4. Then, after the leading left-moving edge approaches the left wall, the current continues to propagate during a transient stage to the fina stage. During the last stage, the height of the nose increases and the current has a thin tail which decreases with time. The most volume of the current is concentrated in the domain of the head. However, the behavior of the nose is peculiar: the front remains almost at constant height $h_{N}$ and speed $u_{N}$. Indeed, Fig. 8 shows that from $t \approx 5.5$ until $\approx 10.5$, the height of the nose $h_{N}$ is close to $\approx 0.66$ and its speed $u_{N}$ is close to $\approx 1$.

Similar behavior was observed for $\theta=15^{\circ}$ (see Fig. 9). During the last propagation stage, the height and the velocity of the nose are almost constant; however, the value of $h_{N}$ is greater by about $30 \%$ when compared to the one for $\theta=10^{\circ}$, while the velocity of the nose in both cases is very close to 1 and differs by less than $10 \%$.

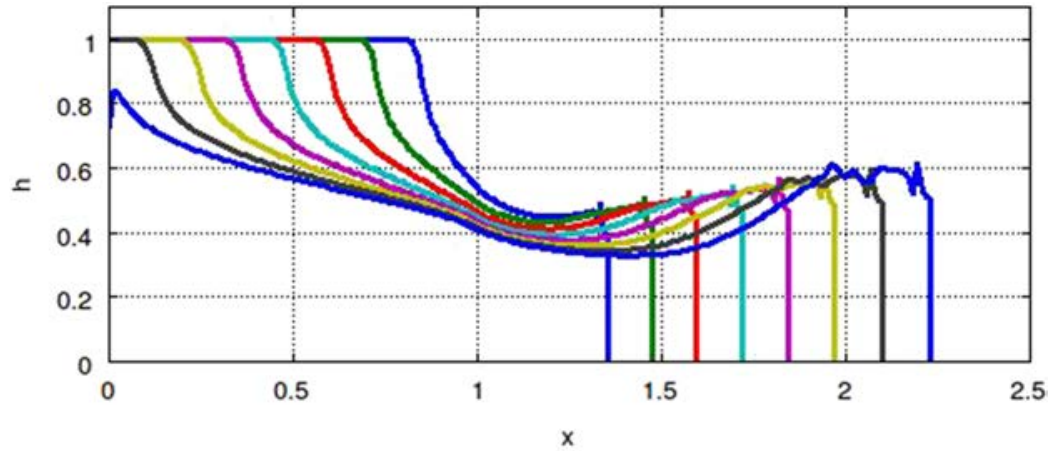

(a)

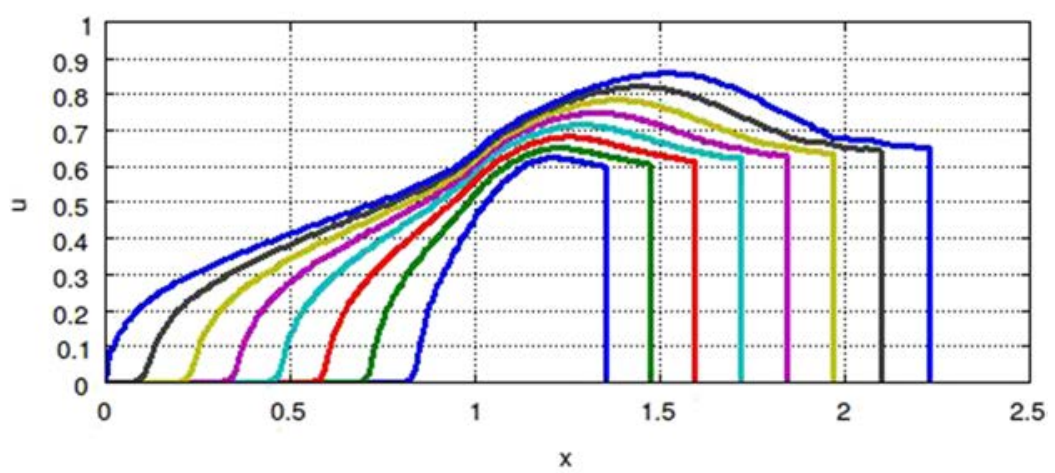

(b)
FIG. 7. SW model results in the downslope case: (a) $h$ and (b) $u$ as functions of $x$ at various $t=0.4(0.2) 1.8$. Here, $\theta=15^{\circ} ; X_{1}=1 ; H_{0}=1.2$. 


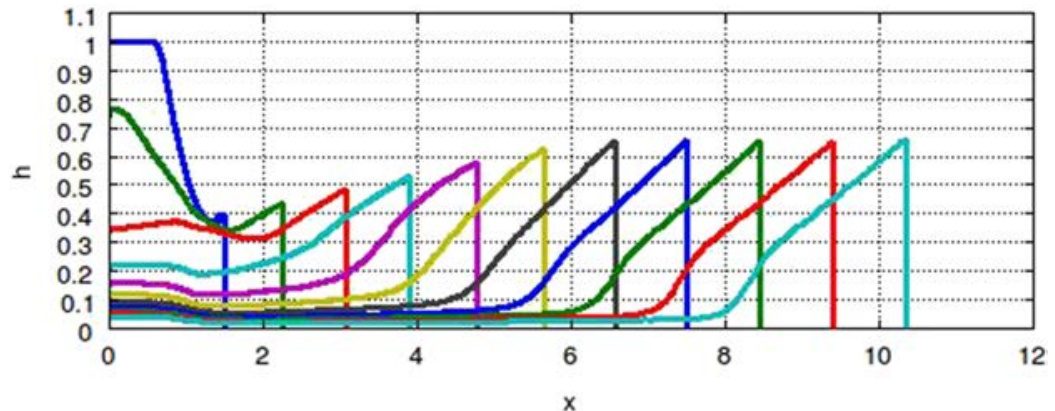

(a)

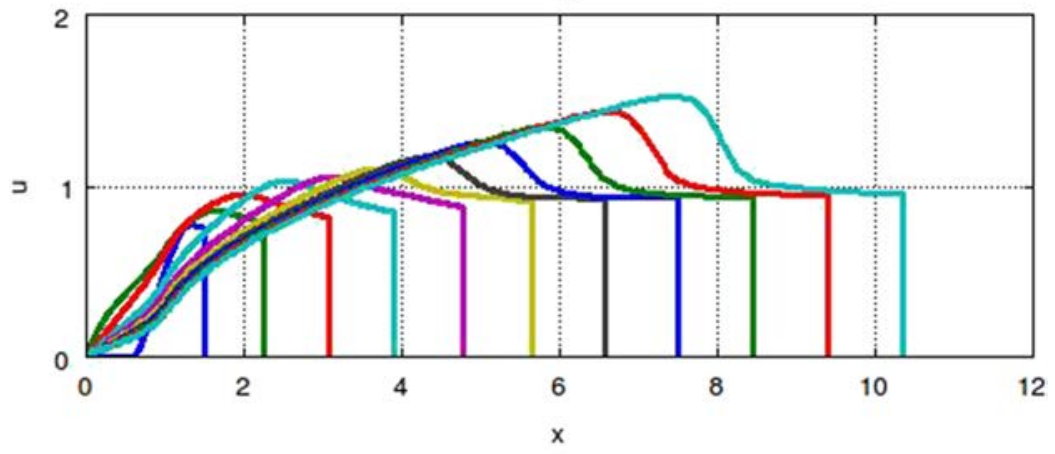

(b)

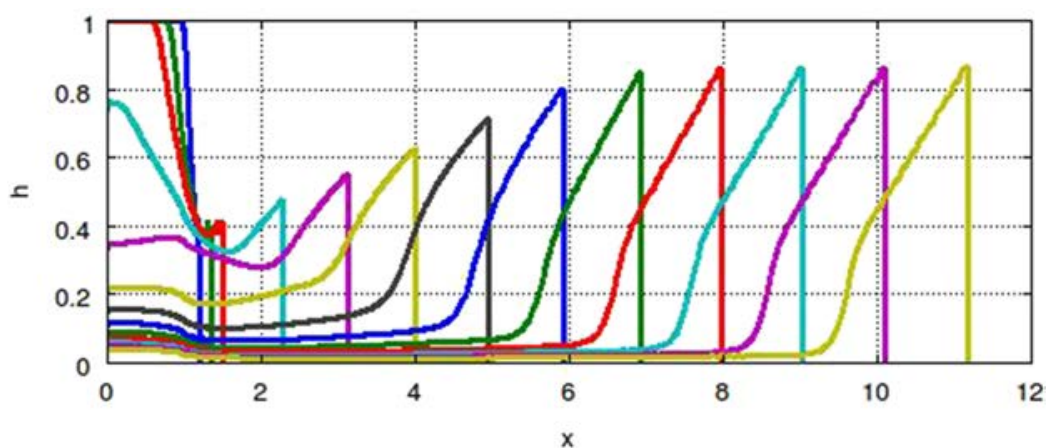

(a)

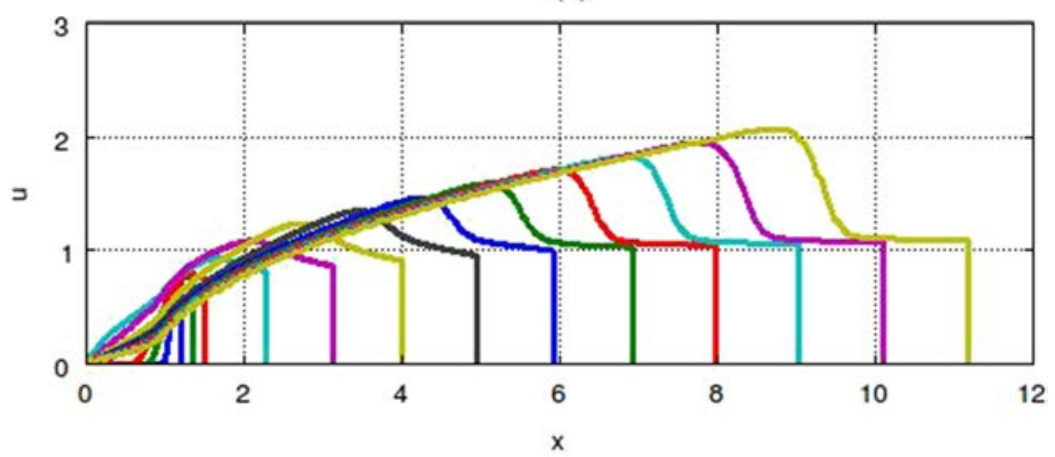

(b)
FIG. 8. SW model results in the downslope case: (a) $h$ and (b) $u$ as functions of $x$ at various $t=0.5(1.0) 10.5$. Here $\theta=10^{\circ} ; X_{1}=1 ; H_{0}=3$.

FIG. 9. SW model results in the downslope case: (a) $h$ and (b) $u$ as functions of $x$ at various $t=0.5(1.0) 10.5$. Here, $\theta=15^{\circ} ; X_{1}=1 ; H_{0}=3$. 


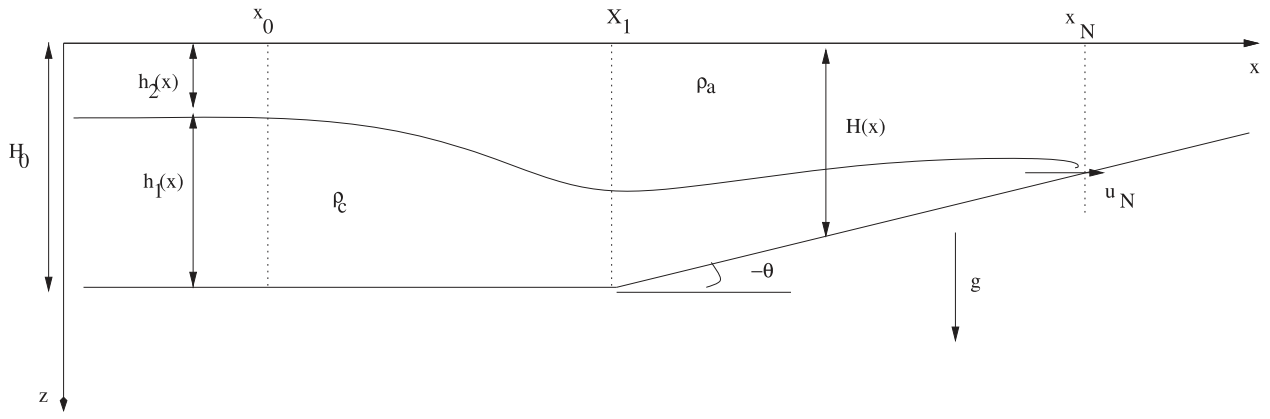

FIG. 10. Schematic description of the current released from a lock of length $x_{0}$ and height $h_{0}$ in the upslope bottom system of height $H(x)$.

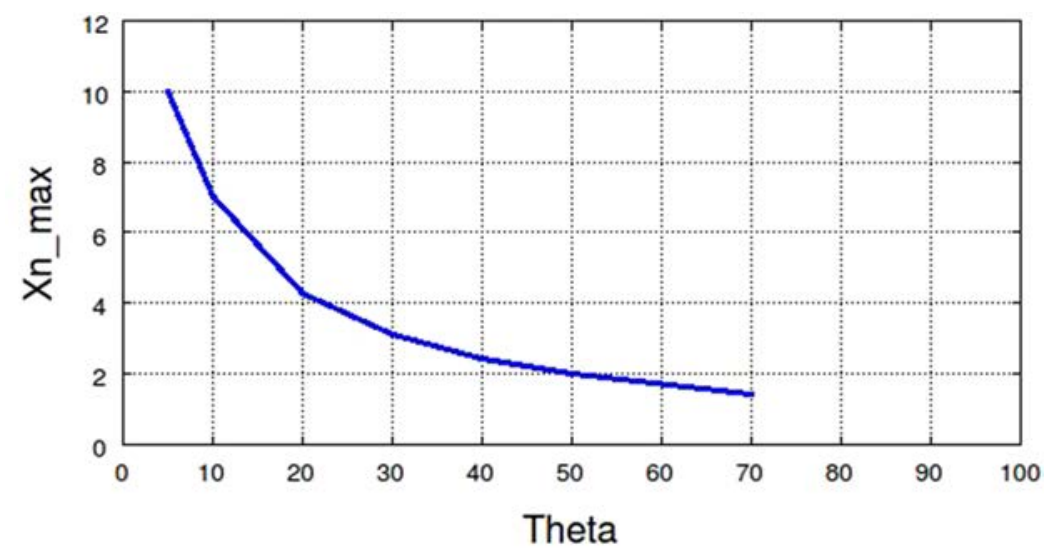

(a)

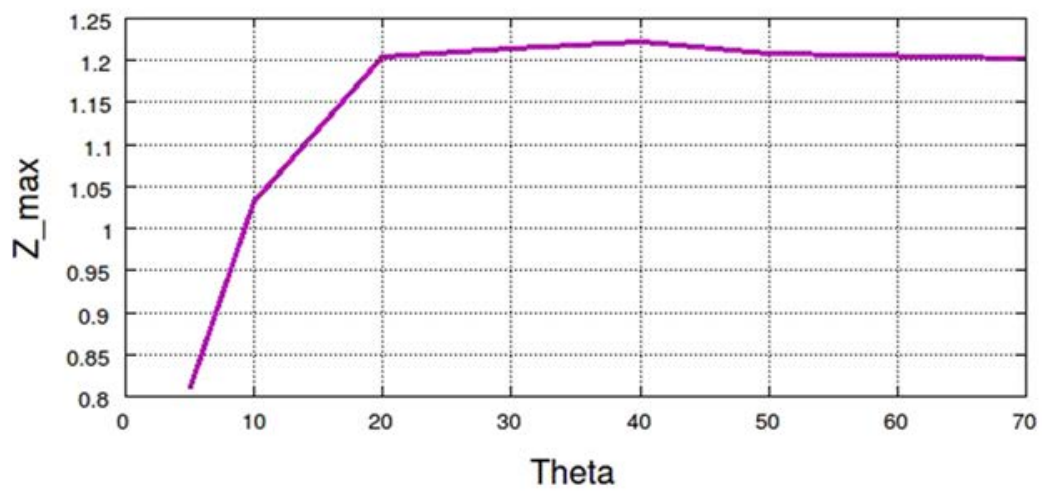

FIG. 11. Upslope case: (a) maximal distance of propagation $x_{N_{\max }}$ vs $-\theta$ and (b) maximal height $Z_{\max }$ vs $-\theta$.

(b)

\section{B. Upslope bottom, $\theta<0$}

The system configuratio for this case is shown in Fig. 10.

Gravity currents propagate up on the slope and are expected to approach the maximal distance of propagation $x_{N_{\max }}$ at height $Z_{\max }$ at some time $t_{\text {stop }}$ and to stop. Figure 11 shows results obtained for $H_{0}=3.0 ; R=1 ; X_{1}=1 ; x_{0} / h_{0}=1$. In Fig. 11(a), the maximal distance approached by the current is plotted as function of $|\theta|$ : the current propagates to longer distances as $|\theta|$ decreases and stops already at the beginning of the slope for quite large values of $|\theta|(|\theta| \geq 70)$. The maximal height approached by the current is also shown in Fig. 11(b): first the height increases with $|\theta|$, and for $|\theta| \approx 20^{\circ}$, it approaches its maximum $Z_{\text {max }} \approx 1$.2. Larger values of $|\theta|$ do not change this maximum of $Z_{\max }$.

\section{COMPARISON WITH EXPERIMENTS}

\section{A. Upslope flow}

We made comparisons with the recent experiments of Ref. 21. The system was Boussinesq of salt water in fresh water. Currents 
were released from horizontal locks, propagated on the horizontal bottom for a while (in the classical slumping phase), and then climbed on slopes of $14^{\circ}-49^{\circ}$. During the upslope motion, $u_{N}$ decelerates and stops at the maximal height $Z_{\max }$ (measured from the bottom). The measured $Z_{\text {max }} / h_{0}$ increases slightly with $H(0)$ (the height ratio of the channel to the lock). The SW equations reproduce well these observations: constant $u_{N}$ up to the beginning of the slope at $X_{1}$ than continuous deceleration to $u_{N}=0$.

Figure 12 shows a comparison between the SW prediction and experimental data for (a) $H(0)=1.33$ and (b) $H(0)=2.0$. We think that the agreement is good. The SW results overpredict the length of propagation by less than $10 \%$. This type of discrepancy has been reported also for currents in horizontal propagation (e.g., Refs. 26, 19, and 30). This can be attributed to viscous friction at the bottom, which is expected to be more pronounced in domains with decelerated fluid

An interesting observation of Ref. 21 is that the GC on the up-slope domain maintains a nearly self-similar shape during propagation. This observation is compared with the predictions of the SW model in Fig. 13. There is fair agreement. The discrepancies can be attributed to (1) the angle $\theta$ is large and (2) during the upslope motion, the speed decreases and the effect of viscous forces increases.

If the up-slope bottom is truncated by an abrupt descent before the point of $u_{N}=0$, a part of the dense flui will be drained over this edge (top), and the other part will develop reverse flow This effect, called overtopping in geophysical applications, is governed by a free critical $u=\left(g^{\prime} h\right)^{1 / 2}$ condition at the edge, resembling the drainage of a reservoir discussed in Ref. 23. The truncated upslope configuratio is out of the scope of the present study, and the interested reader is referred to Ref. 14 (note that the non-Boussinesq $\rho_{a} / \rho_{c}=0$ case is considered there).

The observed agreement/disagreement between the model and experiments is the same as in classical horizontal configuration considered in the literature, e.g., Refs. 26, 17, 19, and 30. The conclusion is that the SW theory is a valuable approximation for calculating the propagation of the current in a configuratio with an up-slope.

\section{B. Downslope case}

The downslope current turns out to be a more challenging topic. For clarifications we performed comparisons with the

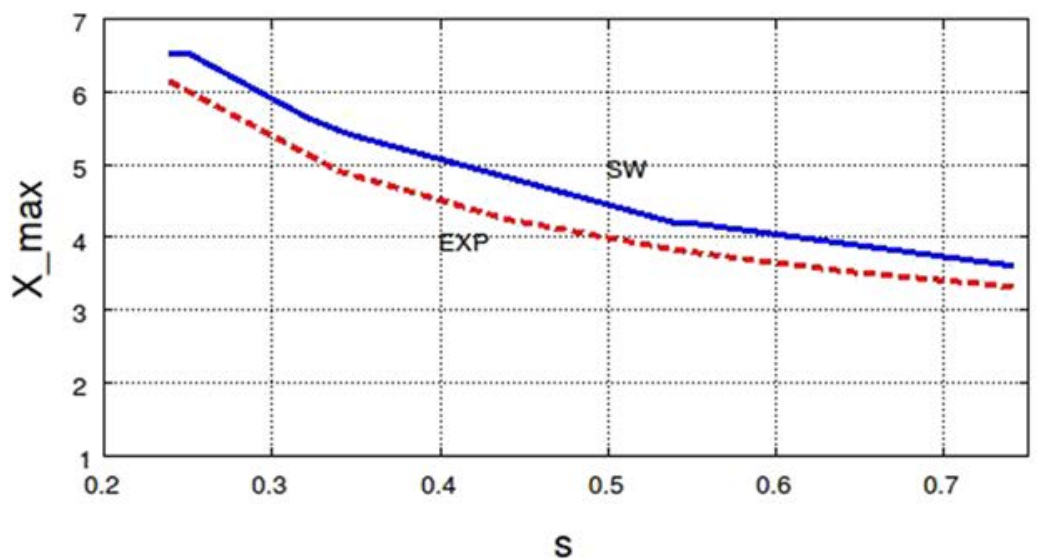

(a)

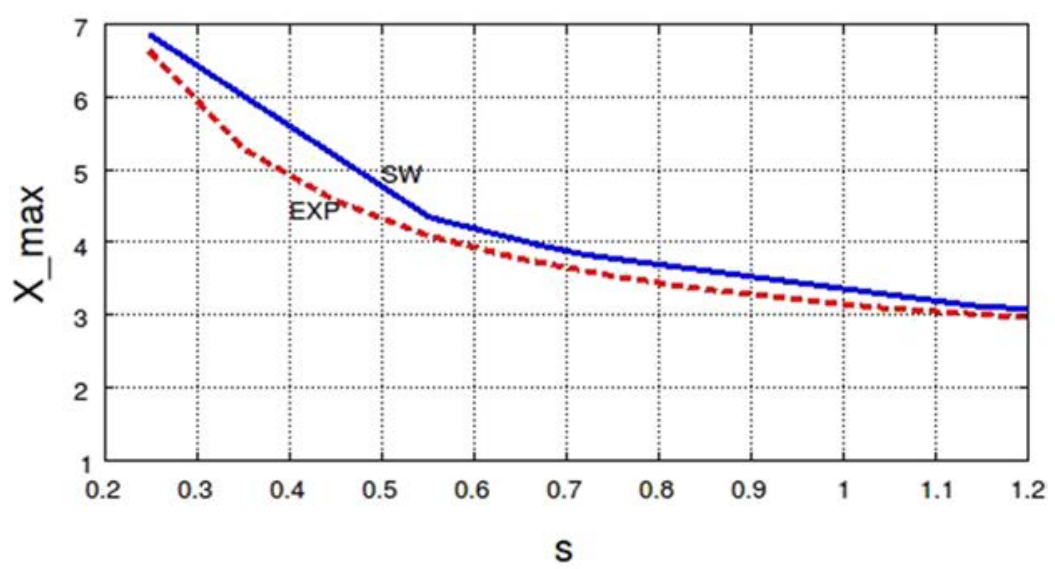

(b)
FIG. 12. Comparison of maximal distance of propagation in the upslope case as a function of slope $S=\tan (-\theta)$ : SW numerical solution (solid line) and experimental results (dashed line) for (a) $H_{0}=1.33$ and (b) $H_{0}=2$. 


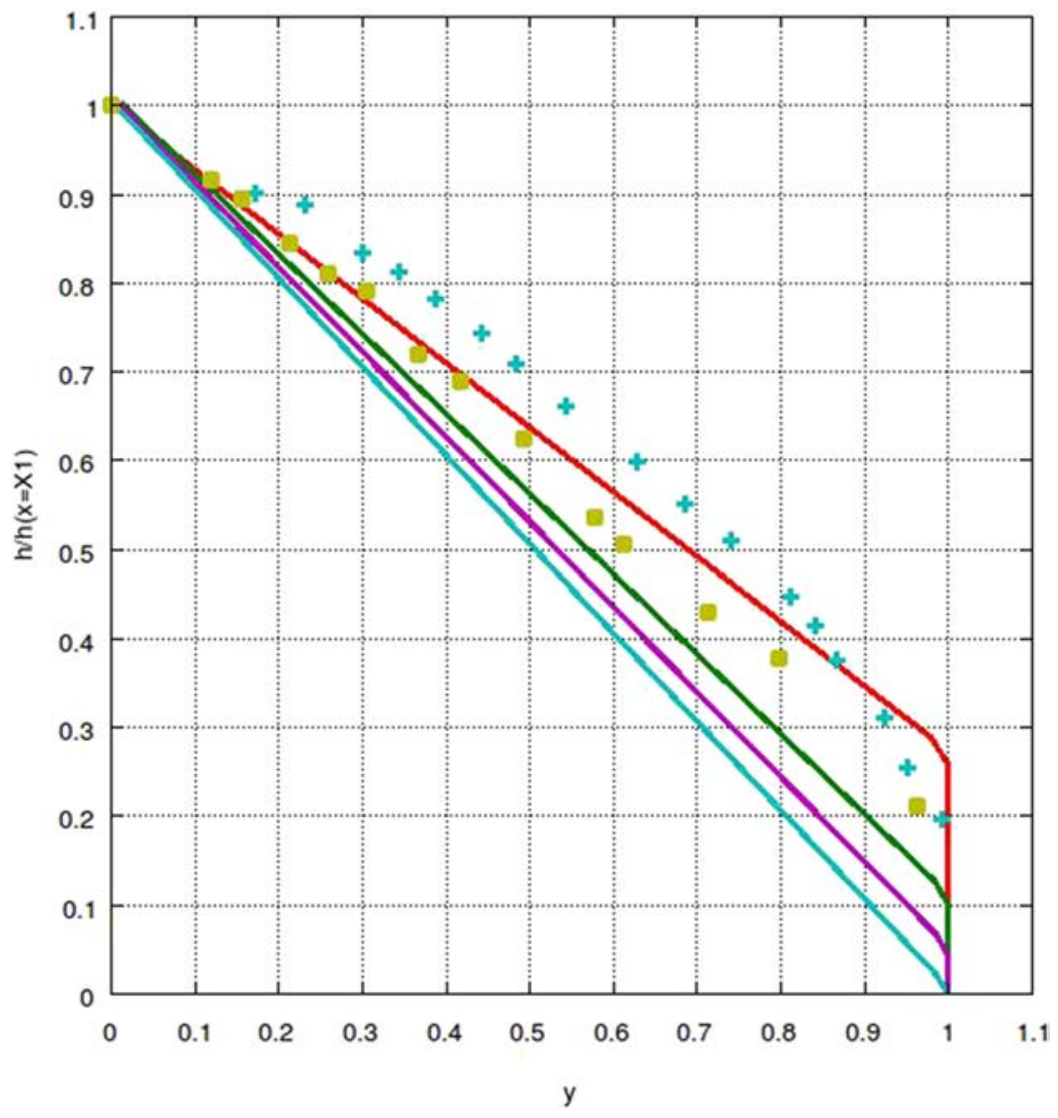

FIG. 13. Shape of GC on the upslope domain, $\theta=-47.7^{\circ}$. A comparison of experiment ${ }^{21}$ [Fig. 5(e)] (symbols) with SW theory (lines): $\mathrm{t}=2.3$ (red), 2.9 (green), 3.3 (purple), and 3.9 (cyan). experiments listed in Table I. The system was of salt solution in fresh water, in a tank of about $250 \mathrm{~cm}$ long and $60 \mathrm{~cm}$ height. These experiments cover a range of $\theta$ and aspect ratio of $x_{0} / h_{0}$, mostly in the Boussinesq domain; the last three experiments in the table are in the slightly non-Boussinesq domain $\rho_{c} / \rho_{a} \approx 1.17$ (attained with sodium chloride solution in fresh water).

Figure 14 shows the data of $x_{N}$ vs $t$ for the Boussinesq experiments, in the dimensionless form. The cases with $\theta \in\left[5.9^{\circ}, 9^{\circ}\right]$ tend

TABLE I. List of downslope experiments used for comparison. M denotes Ref. 22, D denotes Ref. 8, and Dnb denotes Ref. 9. The figure number in the appropriate paper is given in Remark. The units are cgs.

\begin{tabular}{lcccccc}
\hline \hline Label & $\theta$ & $x_{0}$ & $h_{0}$ & $x_{0} / h_{0}$ & $g^{\prime}$ & Remark \\
\hline M1 & 5.9 & 5.5 & 2.5 & 2.2 & 78.7 & 17 \\
M2 & 10.6 & 20.01 & 9.7 & 2.06 & 11.58 & 4 \\
M3 & 10.6 & 5.5 & 9.7 & 0.67 & 99.57 & 12 \\
D1 & 2 & 10 & 8 & 1.25 & 17.02 & 11 \\
D2 & 6 & 10 & 8 & 1.25 & 17.11 & 9 \\
D3 & 9 & 10 & 8 & 1.25 & 17.11 & 4 \\
Dnb1 & 2 & 10 & 8 & 1.25 & 167.1 & 10 \\
Dnb2 & 6 & 10 & 8 & 1.25 & 166.7 & 8 \\
Dnb3 & 9 & 10 & 8 & 1.25 & 167.1 & 5 \\
\hline \hline
\end{tabular}

to collapse on the same curve. Some data cover short propagation $\left(x_{N} \approx 12\right)$, and some cover long propagation $x_{N} \approx 42$. The reason is the limitation of the experimental tank. The available run of the slope was $L \approx 240 \mathrm{~cm}$, and hence, the maximum dimensionless $x_{N}$ is fixe by $L / x_{0}$. In general, the curves show a significan deceleration of the current starting at $x_{N} \approx 8-10$ (the $\theta=2^{\circ}$ case displays the strongest deceleration).

A comparison of the SW results with the experimental data shows agreement for short propagation only, roughly $x_{N}<5-10$; afterward, the experimental $x_{N}(t)$ is significantl smaller. A typical result is shown in Fig. 15.

A closer inspection reveals an essential qualitative discrepancy: the experimental speed of propagation $u_{N}$ has an accelerationdeceleration pattern, while the SW $u_{N}$ lacks the deceleration phase. This indicates that a physical effect is missing in the SW modeling of the downstream current. The plausible candidates are entrainment and drag.

\section{SWE model with entrainment and drag}

The question is why are the entrainment and drag necessary in the downslope configuratio while fairly negligible in the upstream case. Our explanation is as follows. There is solid evidence that the entrainment effect is associated with an interfacial instability. ${ }^{18,25}$ The relevant parameter is the Richardson number which is approximated by the "bulk" value $R i=g^{\prime} h^{*} /\left(u^{*}\right)^{2}$ (the asterisk denotes 


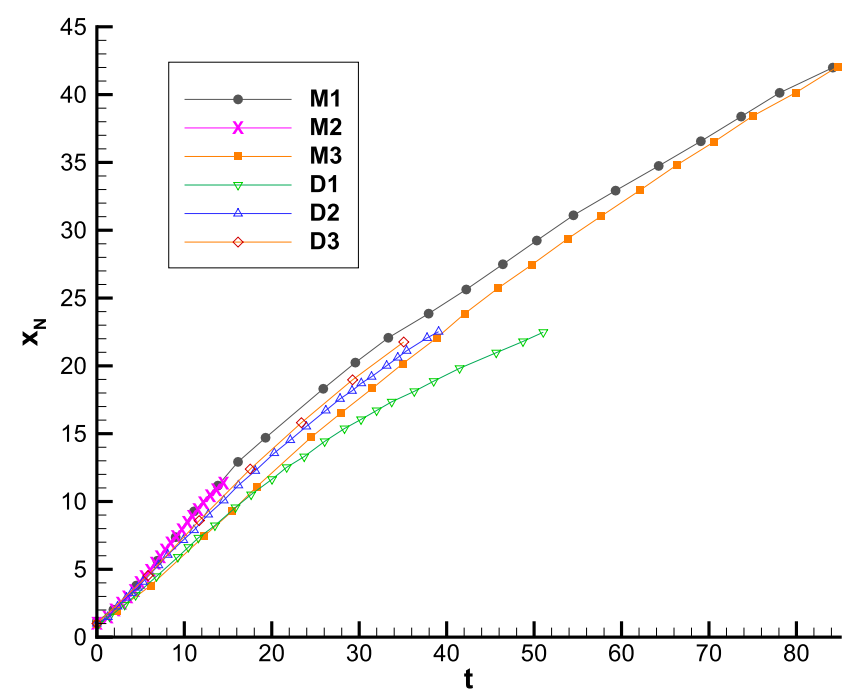

FIG. 14. $x_{N}$ vs $t$ experimental data for Boussinesq downslope configurations $M 1$, $M 2, M 3, D 1, D 2, D 3$ of Table I.

dimensional variables). For $R i>R i_{\text {crit }} \approx 0.5$, the interface is expected to be stable, but for smaller values of $R i$, Kelvin-Helmholtz vortices appear, causing both entrainment/mixing of ambient flui into the current and drag. In the horizontal flow $R i \approx 1 / F r^{2} \sim 1$. In the up-slope flow $u$ decreases during propagation, and hence, $R i$ increases. Consequently, the SW solution with no entrainment is a fair approximation to these flows

The downslope flo is different. The speed of the nose $u_{N}$ increases, while $h$ in the tail behind is bound to decrease because of volume continuity, as shown in Figs. 8 and 9. Small values of $R i$ are bound to appear after a modest downslope propagation (5 lock lengths, say). The simple SW equations, which assume a sharp stable smooth interface, become invalid. We therefore apply an extension.

We employ the model suggested by Ref. 18. The entrainment of the upper flui into the current is represented by the flu (speed) $W_{e}(x, t)=E|u(x, t)|$, where $E$ is the entrainment coefficien given by the empirical formula

$$
E=\frac{E_{0}}{1+\psi R i},
$$

where $E_{0}=0.078$ and $\psi=27$. The entrainment is accompanied by a drag term $C_{d} u|u|$, where the drag coefficien is a constant of the order 0.1 when $R i<1$ and zero otherwise. To proceed, we introduce additional simplifications the system is Boussinesq, and the current is deep. Also, the entrained flui is rapidly mixed across the thickness of the layer so that instead of $\rho_{c}$, the current has the diluted density $\rho_{d}(x, t)<\rho_{c}$; consequently, the effective reduced gravity is $g_{e}^{\prime}=\left(\rho_{d} / \rho_{a}-1\right) g$.

The equations for the volume of the current, momentum of the current, and mass of the dense component (represented by $g_{e}^{\prime}$ ), in the dimensional form, are

$$
\begin{gathered}
\frac{\partial h}{\partial t}+\frac{\partial}{\partial x}(u h)=E|u|, \\
\frac{\partial}{\partial t}(u h)+\frac{\partial}{\partial x}\left(u^{2} h+g_{e}^{\prime} \frac{1}{2} h^{2}\right)-g_{e}^{\prime} h \tan \theta=-C_{d} u|u|, \\
\frac{\partial g_{e}^{\prime} h}{\partial t}+\frac{\partial}{\partial x}\left(g_{e}^{\prime} u h\right)=0 .
\end{gathered}
$$

The calculation of $g_{e}^{\prime}(x, t)$ is a part of the problem.

In the characteristic form, this becomes

$$
\left[\begin{array}{c}
h \\
u \\
g_{e}^{\prime}
\end{array}\right]_{t}+\left[\begin{array}{ccc}
u & h & 0 \\
g_{e}^{\prime} & u & \frac{1}{2} h \\
0 & 0 & u
\end{array}\right]\left[\begin{array}{c}
h \\
u \\
g_{e}^{\prime}
\end{array}\right]_{x}=\left[\begin{array}{c}
E|u| \\
g_{e}^{\prime}\left[\tan \theta-\left(C_{d}+E\right) \frac{u|u|}{g_{e}^{\prime} h}\right] \\
-g_{e}^{\prime} E \frac{|u|}{h}
\end{array}\right] .
$$

The system is hyperbolic. The relevant eigenvalues are

$$
c_{ \pm}=u \pm \sqrt{h g_{e}^{\prime}}, \quad \lambda_{3}=u .
$$

The presence of the source terms on the RHS of (4.5) introduces a complex dependency on $t$ along the $c_{ \pm}$characteristics, which defie clear-cut conclusions. The third characteristic is decoupled: along $d x / d t=u, d\left(g_{e}^{\prime}\right)=-g_{e}^{\prime} E|u| d t / h$.

The RHS term of the momentum equation merits attention. Assuming $u>0$, we rewrite $u|u| /\left(g_{e}^{\prime} h\right)=1 / R i$, and hence, the

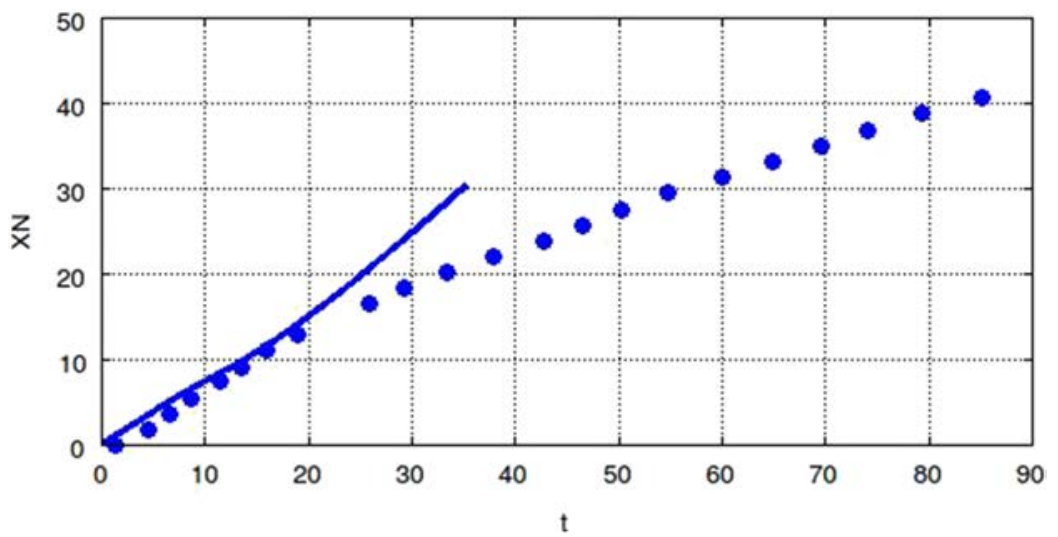

FIG. 15. $x_{N}$ vs $t$ in the downslope configuration of experiment $\mathrm{M} 1\left(\theta=5.9^{\circ}\right)$. SW solution (solid line) and experimental data (dots). 
forcing term is $\propto\left[\tan \theta-\left(C_{d}+E\right) / R i\right]$. The insight is that drag and entrainment combine to oppose the downslope reduced gravity acceleration, and the magnitude of the opposition increases when $R i$ decreases. In other words, the effect of $R i$ is twofold: it controls the appearance of the significan entrainment and drag coefficient due to interface instability, but also attempts to slow-down the motion and stabilize it (a smaller $u$ means a larger $R i$ ). The presence of such a complex mechanism in our simple model must be treated with care and subject to further verification

The front condition is $u_{N}=\operatorname{Fr}\left(g_{e N}^{\prime} h_{N}\right)^{1 / 2}$. The usual $\operatorname{Fr}(a)$ can be used when $\theta, E$, and $C_{d}$ are small. This is justifie by estimating the contribution of those effects in the control-volume derivation of $F r$. Due to the entrainment, $g_{e}^{\prime}$ decreases and $h$ increases; therefore, the slow-down of $u_{N}$ due to entrainment may be small even during a significan dilution of $g_{e}^{\prime}$.

This set of equations, supplemented by the closure (4.1), is referred to as the SW extended (SWE) model.

The standard SW formulation is given by $E=C_{d}=0$, with the front condition $u_{N}=\operatorname{Fr}(a)\left(g^{\prime} h_{N}\right)^{1 / 2}$, where $F r$ is given by HS or Benjamin's equation. The characteristics are

$d u=\mp 2 d\left(g^{\prime} h\right)^{1 / 2}+g^{\prime} \tan \theta d t, \quad$ on $\frac{d x}{d t}=c_{ \pm}=u \pm\left(g^{\prime} h\right)^{1 / 2}$.

The finite-differenc solutions of the one-layer model are similar to those of the two-layer model when the depth ratio $H$ is larger than 1.5, approximately. The results show (e.g., Fig. 8) that the simple SW current accelerates during the downslope flo and forms a prominent head followed by a thin tail. $u_{N}$ attains a maximum of about $0.8\left(g^{\prime} h_{0}\right)^{1 / 2}$ and then maintains this speed within small oscillations. Inspection of the characteristics indicates that the current cannot decelerate. Consider the behavior of $R i=g^{\prime} h / u^{2}$. The value near the nose is about $1 / F r^{2} \approx 0.8$. However, in the tail, $h$ is small due to the spread-out of the fixe volume, and small values of $R i$ appear.

The experiments (see Refs. 22 and 8 and others cited within) confir the initial acceleration and formation of the head-tail shape. However, the realistic current, after attaining a maximum speed of about $0.8\left(g^{\prime} h_{0}\right)^{1 / 2}$, enters into a phase of deceleration. When the deceleration appears the ratio of inertial to viscous effects is still large, and hence, this slow-down effect must be attributed to the appearance of entrainment and drag.

To model this behavior, the authors of Ref. 3 developed a momentum-integral analysis for the propagation, assuming that the motion is governed by a head of self-similar shape that contains a fixe amount of the buoyancy, counteracted by entrainment and drag with constant coefficients The result is, in the dimensional form,

$$
x_{N}=\xi_{0}+K\left(g^{\prime} h_{0} x_{0}\right)^{1 / 3}\left(t+\tau_{0}\right)^{2 / 3},
$$

where $\xi_{0}, \tau_{0}$ are adjustable constants and the dimensionless $K$ is given by a formula which contains $E, C_{d}$ and some adjustable shapefactors. Here, $g^{\prime}$ is the value in the lock. We refer to (4.8) as the power-law model. This curve has been fitte well to various experimental data (e.g., Refs. 22 and 8). The qualitative insight is useful: the downslope current with entrainment and drag displays, after a while, $x_{N} \sim t^{2 / 3}$ like a standard horizontal CG in the self-similar stage. However, this is a not a reliable prediction tool because (1) the fact that no front condition can be applied and the assumptions of a selfsimilar head and constant $E, C_{d}$ lack theoretical justificatio and (2) adjustable constants are necessary. In particular, the value $K$ in the experiments $(\approx 2.7)$ differs significantl from theoretical evaluations. (3) Moreover, this model is relevant only during the deceleration stage.

This bring us to the suggestion that the SWE model is a more efficien tool. The disadvantage is that the solution must be calculated numerically. The advantage is that the SWE model is valid for both the acceleration and deceleration stages. The question is if we can achieve accurate predictions without adjustable parameters. The tests performed here provide support to the suggestion.

Figure 16 illustrates the SWE predictions for configuratio D3 of Table I $\left(\theta=9^{\circ}\right)$. The dilution is represented by $\alpha=g_{e}^{\prime} / g^{\prime}$ given in the third panel. We see that the shape $h(x)$ is triangular, and the most significan dilution occurs in the tail, while the buoyancy of the domain behind the nose decreases more slowly (to about $60 \%$ at $t=40$ ). This is consistent with the observations in the experiments, but a quantitative comparison cannot be performed with the available data. The bulk Richardson number $R i$ decreases during the propagation, and this sustains the deceleration effect.

The available experimental data allow for a detailed comparison for the propagation. In Fig. 17, $x_{N}(t)$ is compared with experimental data reported by Ref. 8. $x_{N}(t)$ of the simple SW model $\left(E_{0}=C_{d}\right.$ $=0)$ is in fair agreement with the measurements during some distance of propagation and then overestimates. The SWE model reproduces well the observed acceleration-deceleration pattern. According to this model, the volume increase due to entrainment is $7 \%$ at $t=10,25 \%$ at $t=10$, and $56 \%$ at $t=30$. The predicted profile of $h, u$, and $\alpha=g_{e}^{\prime} / g^{\prime}$ make sense. Qualitatively, they are in agreement with experimental observations, but a qualitative comparison is not possible because of the lack of dedicated data.

Figures 18-21 show comparisons of propagation for the other configuration listed in Table I. We note that the overall agreement for the Boussinesq systems is fair. We must keep in mind that in general SW predictions (even for the simplest horizontal lock-release problem) deviate by $10 \%-20 \%$ from data. In the downslope config uration, there is big sensitivity to the initial conditions because they may affect the instability of the interface. Indeed, the authors of Ref. 22 reported "differences in velocity history for similar initial parameter values" (see Fig. 5 in that paper). Consequently, some of the discrepancies may be attributed to the fact that laboratory experiments contain a built-in uncertainty concerning the Richardson number. The non-Boussinesq experiments show, systematically, a delay in the initial propagation. However, the subsequent $x_{N}(t)$ displays the same behavior as in the Boussinesq case. This delay requires further investigation. The top of the tank was open to the atmosphere, and in non-Boussinesq systems, this introduces differences with the fixed-to assumed by the theory.

We argue that the extended SW model performs well. We admit that the entrainment and drag components lack rigor. However, we claim that our model is more advantageous as compared to the widely used model of Ref. 3. The firs advantage is practical: the SWE model (1) predicts the propagation of the current from given initial conditions without the need for adjustments of constants [the entrainment and drag functions are "off the shelf," and the same (for the parameters tested in this paper, at least)] and (2) is valid for both the acceleration and deceleration phases. The power-law model 

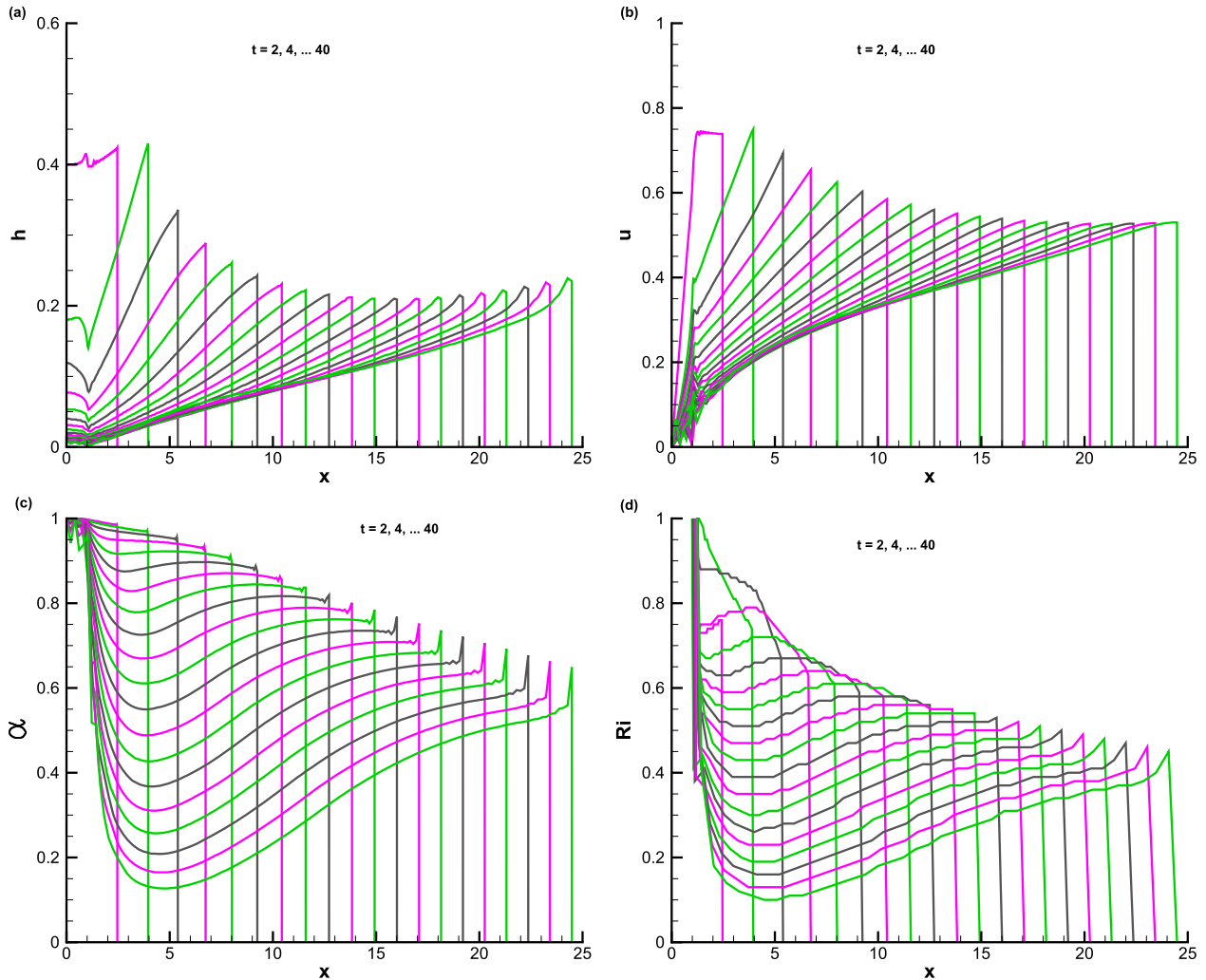

FIG. 16. SWE predictions for the downslope configuration D3 $\left(\theta=9^{\circ}\right)$. Profiles of (a) $h$, (b) $u$, (c) $\alpha=g_{\mathrm{e}}^{\prime} / g^{\prime}$ and (d) $R i$ as functions of $x$ at various times $t=2,4$, ..., 40 . needs adjustable $\xi_{0}, \tau_{0}$ which depend on $\theta, g^{\prime}$, and $x_{0} / h_{0}$; then, it applies only to the deceleration phase, starting at some $t_{1}$ which must also be determined empirically. The second advantage is conceptual: the downslope gravity current is the same flow-fie manifestation

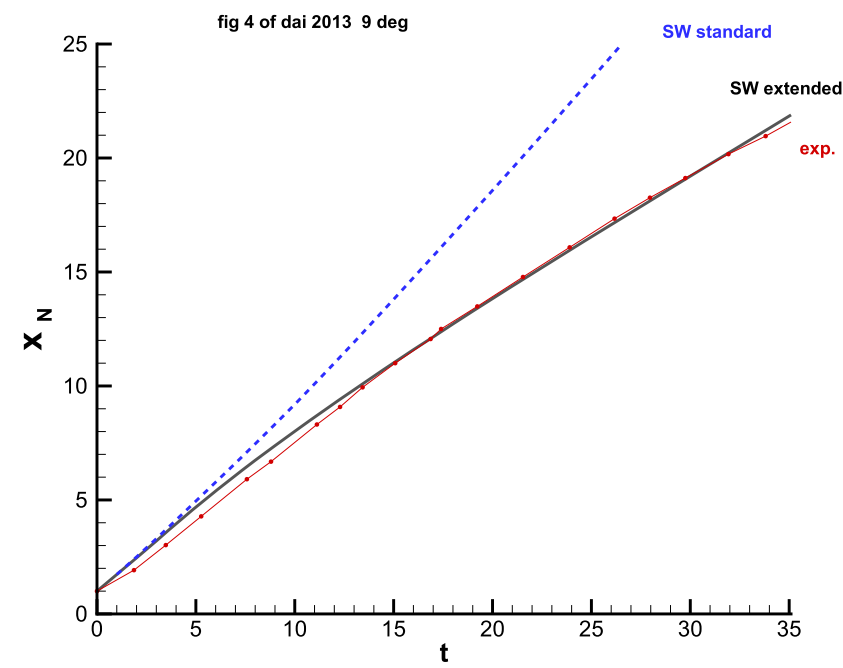

FIG. 17. $x_{N}$ vs $t$ : experimental data and predictions of $S W$ standard $\left(E=C_{d}=0\right)$ and SWE models. Downslope configuration D3 $\left(\theta=9^{\circ}\right)$. like the counterparts on a horizontal bottom or upslope bottom, and the difference is a clear-cut result of the internal $\operatorname{Ri}(x, t)$. The power-law model pictures the downslope current as a semicircular patch which has no counterpart in the accepted theory for the

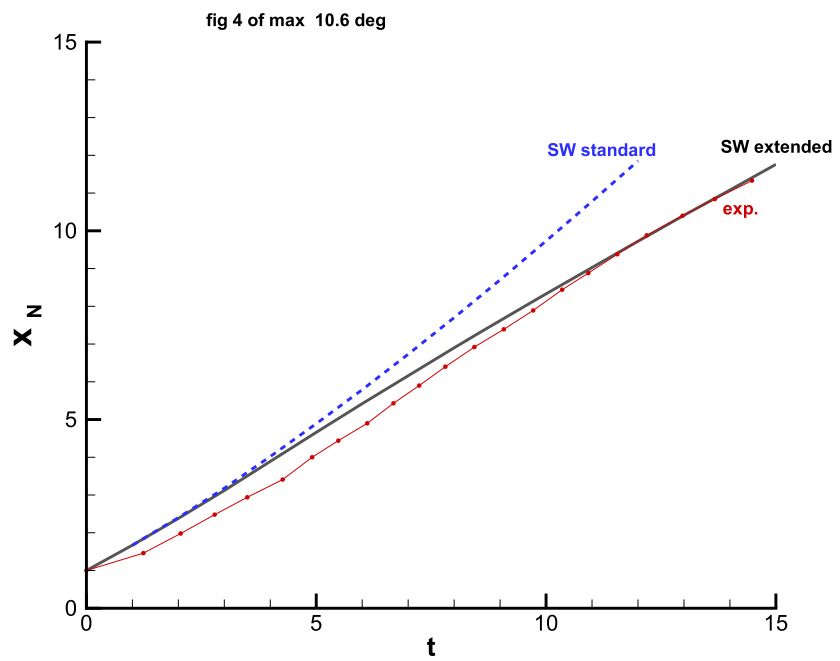

FIG. 18. $x_{N}$ vs $t$ for downslope configuration M2 $\left(\theta=10.6^{\circ}\right)$. Note the short propagation because this experiment was with long $x_{0}=20 \mathrm{~cm}$. 


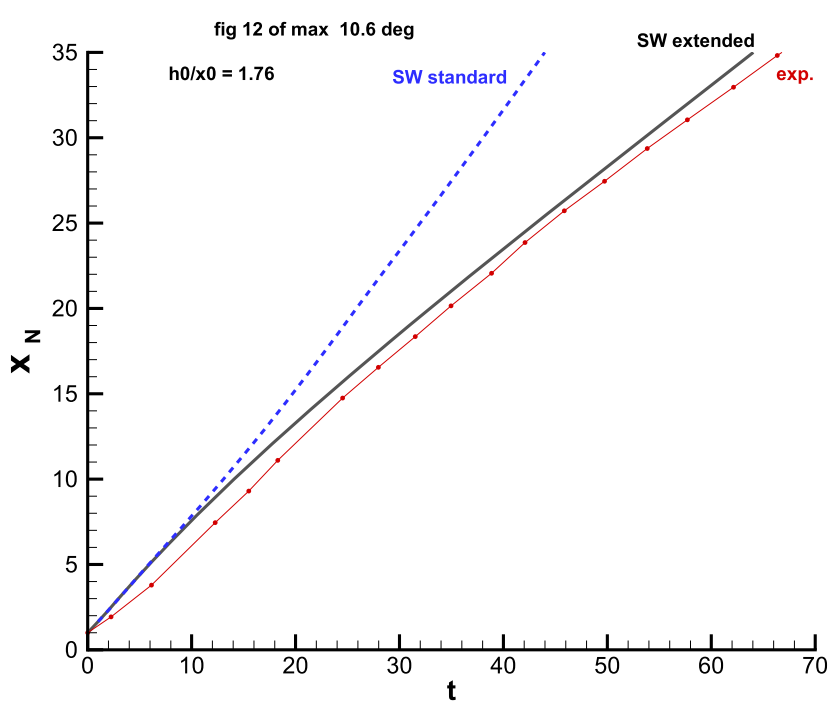

FIG. 19. $x_{N}$ vs $t$ for downslope configuration M3 $\left(\theta=10.6^{\circ}\right)$. Note the long propagation because this experiment was with short $x_{0}=5.5 \mathrm{~cm}$.

horizontal gravity current $\left(x_{N} \sim t^{2 / 3}\right.$ for large $t$ is just a coincidence). The third advantage is concerned with the internal structure of the flow-fiel the SWE model provides $h, u, g_{e}^{\prime} / g^{\prime}$, and $R i$ as functions of $x$, t. Although these are depth-averaged results not accurate pointwise values, they are expected to provide insights into the distribution of the momentum and mixing along the current.

The formal major advantage of the power-law model is the analytical simplicity of the $x_{N}(t)$ formula. However, this is an illusion because this formula cannot be used without experimental measurements of $x_{N}(t)$, and hence, the formula is more a postprocessor than a predictor. Moreover, the finite-differenc solution of the SWE hyperbolic system for $h, u, g_{e}^{\prime}$ as functions of $x, t$ is nowadays a quick computation on a laptop computer.

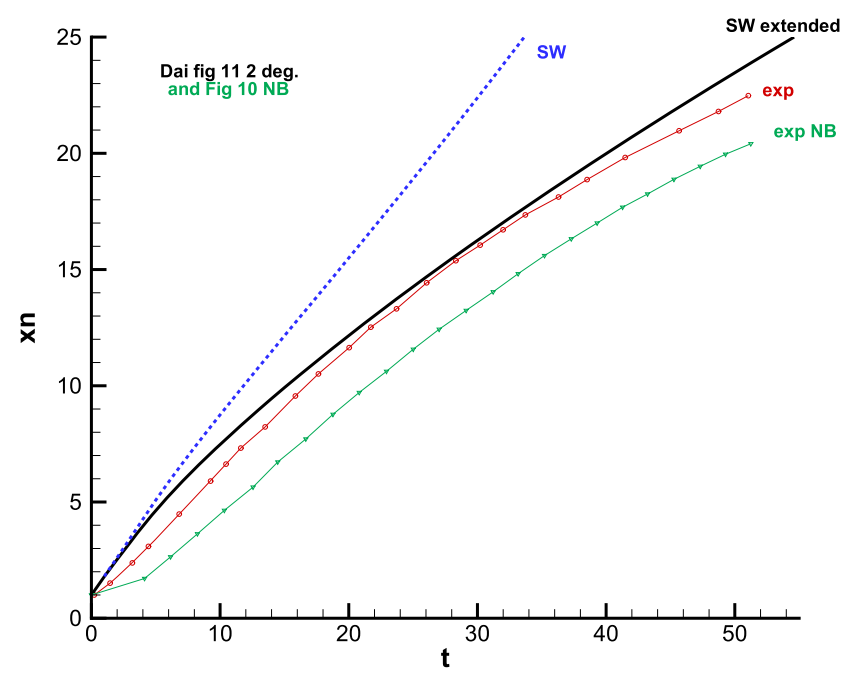

FIG. 20. $x_{N}$ vs $t$ for downslope configurations D1 and Dnb1 $\left(2^{\circ}\right)$.

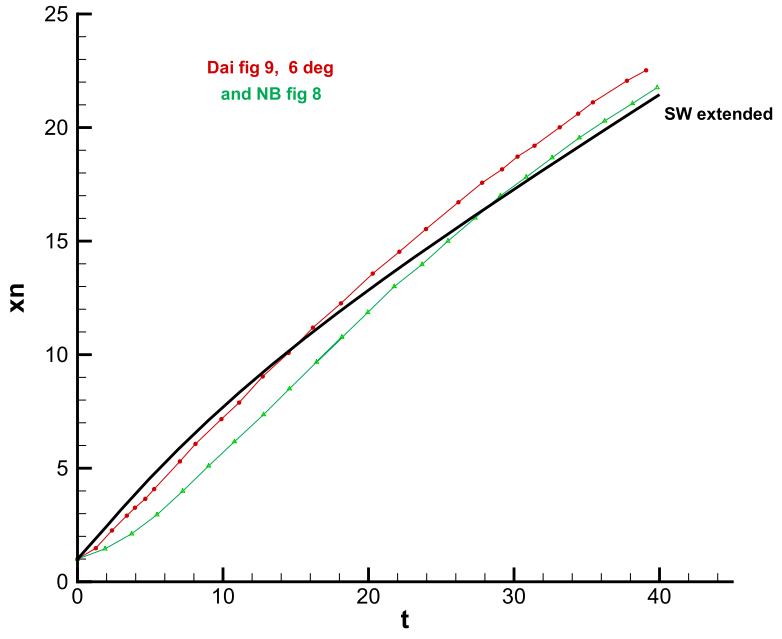

(a)

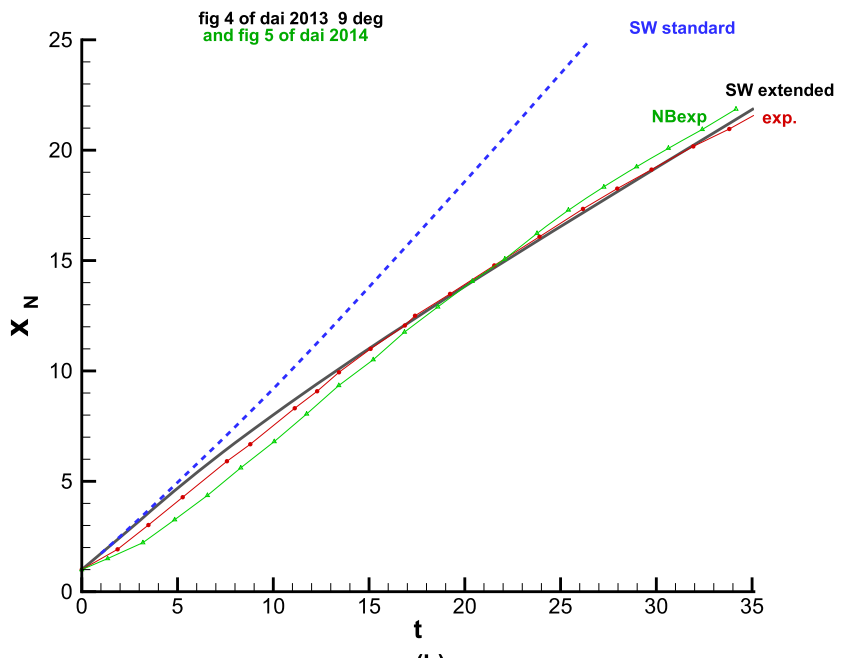

(b)

FIG. 21. $x_{N}$ vs $t$ for downslope configurations (a) D2 and Dnb2 $\left(6^{\circ}\right)$ and (b) D3 and Dnb3 $\left(9^{\circ}\right)$.

To summarize, we think that the analysis of the downslope current should be based on the SWE models (with possible modifi cations) and the power-law model can be dismissed with due credit for it being the backbone of numerous published studies.

\section{CONCLUDING REMARKS}

We have revisited the problem of a gravity current of fixe volume released from a lock, which then encounters a downslope or upslope bottom, in the inertial-buoyancy regime. The analysis uses the shallow-water (SW) approximation. The system of equations is hyperbolic, and a Froude-number jump condition is applied at the nose (front).

The obvious parameter of the problem is the slope angle, $\theta$. However, we elucidated that the propagation of the current is affected by additional parameters: the aspect ratio of the lock $x_{0} / h_{0}$, 
the height ratio of the ambient to lock, $H / h_{0}$, and the distance of the gate from the beginning of the slope. The SW model provides qualitative insights and quantitative data concerning the influenc of these parameters.

We performed comparisons of the theoretical results with previously published experimental data. For the up-slope current, the agreement is satisfactory: both the model and experiment show that the current decelerates up to a point where it stops $u_{N}=0$; the SW predictions slightly overestimate the maximum height.

The down-slope current system displays a different pattern. The SW results indicate that the current accelerates and then $u_{N}$ attains a constant value, $\approx\left(g^{\prime} h_{0}\right)^{1 / 2}$. In experiments, the current firs accelerates and then decelerates. We showed that this can be attributed to the behavior of the Richardson number $R i$ : during the acceleration, $R i$ decreases, and eventually, an instability develops at the interface which produces entrainment/mixing and drag. We demonstrated that when these effects are added to the simple shallow-water balances (using an off-the-shelf closure for the entrainment coefficien and a constant drag coefficient) the shallow water extended model (SWE) indeed predicts an acceleratingdecelerating downslope current, which is in good agreement with previously published experimental data.

We think that, overall, this work improves the understanding and modeling of gravity currents. In particular, we demonstrate that the fact that $R i$ increases in the up-slope case and decreases in the down-slope case is the reason why the simple SW formulation works well in the firs case but needs entrainment and drag extension in the second case. In our opinion, the extended SW model presented in this work is an efficien replacement to the widely used power-law $x_{N}=\xi_{0}+K\left(g^{\prime} h_{0} x_{0}\right)^{1 / 3}\left(t+\tau_{0}\right)^{2 / 3}$ (dimensional) model of Ref. 3 because the latter model (1) uses adjustable constants $\xi_{0}, \tau_{0}$, $K$ and (2) applies only to the deceleration stage. More investigation is still needed to further assess the accuracy of the entrainment and drag closures needed in the extended SW model. This requires careful comparisons with more experimental data. Additional interesting direction is the study by Navier-Stokes simulation which might point out details of the motion (see the work of $\mathrm{Qu}^{29}$ ) and must be left for future work.

\section{REFERENCES}

${ }^{1} \mathrm{P}$. G. Baines, "Mixing in downslope flow in the ocean-plumes versus gravity currents," Atmos.-Ocean 46(4), 405-419 (2008).

${ }^{2}$ P. G. Baines and S. Condie, "Observations and modelling of antarctic downslope flows A review," in Ocean, Ice, and Atmosphere: Interactions at the Antarctic Continental Margin, Antarctic Research Series Vol. 75 (American Geophysical Union, 1998), pp. 29-49.

${ }^{3}$ P. Beghin, E. J. Hopfinger and R. E. Britter, "Gravitational convection from instantaneous sources on inclined boundaries," J. Fluid Mech. 107, 407-422 (1981).

${ }^{4}$ T. B. Benjamin, "Gravity currents and related phenomena," J. Fluid Mech. 31, 209-248 (1968).

${ }^{5}$ V. K. Birman, B. A. Battandier, E. Meiburg, and P. F. Linden, "Lock-exchange flow in sloping channels," J. Fluid Mech. 577, 53-77 (2007).

${ }^{6}$ R. T. Bonnecaze, H. E. Huppert, and J. R. Lister, "Particle-driven gravity currents," J. Fluid Mech. 250, 339-369 (1993).
${ }^{7}$ C. Cenedese, J. Whitehead, T. Ascarelli, and M. Ohiwa, “A dense current flow ing down a sloping bottom in a rotating fluid, J. Phys. Oceanogr. 34(1), 188-203 (2004).

${ }^{8}$ A. Dai, "Experiments on gravity currents propagating on different bottom slopes,” J. Fluid Mech. 731, 117-141 (2013).

${ }^{9}$ A. Dai, "Non-Boussinesq gravity currents propagating on different bottom slopes," J. Fluid Mech. 741, 658-680 (2014).

${ }^{10}$ A. Etrati and I. A. Frigaard, "A two-layer model for buoyant inertial displacement flow in inclined pipes," Phys. Fluids 30(2), 022107 (2018).

${ }^{11}$ H. J. S. Fernando, "Fluid dynamics of urban atmospheres in complex terrain," Annu. Rev. Fluid Mech. 42, 365-389 (2010).

${ }^{12}$ W. George, H. Abrahamsson, J. Eriksson, R. Karlsson, L. Löfdahl, and M. Wosnik, "A similarity theory for the turbulent plane wall jet without external stream,” J. Fluid Mech. 425, 367-411 (2000).

${ }^{13}$ H. P. Greenspan and R. E. Young, "Flow over a containment dyke," J. Fluid Mech. 87(1), 179-192 (1978).

${ }^{14}$ A. J. Hogg, T. E. Baldock, and D. Pritchard, "Overtopping a truncated planar beach," J. Fluid Mech. 666, 521-553 (2011).

${ }^{15}$ E. J. Hopfinger "Snow avalanche motion and related phenomena," Annu. Rev. Fluid Mech. 15(1), 47-76 (1983).

${ }^{16}$ H. E. Huppert, “Gravity currents: A personal perspective,” J. Fluid Mech. 554, 299-322 (2006).

${ }^{17}$ H. E. Huppert and J. E. Simpson, “The slumping of gravity currents,” J. Fluid Mech. 99, 785-799 (1980).

${ }^{18}$ C. G. Johnson and A. J. Hogg, "Entraining gravity currents," J. Fluid Mech. 731, 477-508 (2013).

${ }^{19}$ J. B. Klemp, R. Rotunno, and W. C. Skamarock, "On the dynamics of gravity currents in a channel," J. Fluid Mech. 269, 169-198 (1994).

${ }^{20}$ V. Lombardi, C. Adduce, and G. Sciortino, "Gravity currents flowin upslope: Laboratory experiments and shallow-water simulations," Phys. Fluids 27, 016602 (2015).

${ }^{21}$ L. J. Marleau, M. R. Flynn, and B. R. Sutherland, "Gravity currents propagating up a slope," Phys. Fluids 26(4), 046605 (2014).

${ }^{22}$ T. Maxworthy, "Experiments on gravity currents propagating down slopes. Part 2. The evolution of a fixe volume of heavy flui released from closed locks into a long open channel," J. Fluid Mech. 647, 27-51 (2010).

${ }^{23}$ M. Momen, Z. Zheng, E. Bou-Zeid, and H. A. Stone, "Inertial gravity currents produced by flui drainage from an edge," J. Fluid Mech. 827, 640-663 (2017).

${ }^{24}$ K. W. Morton and D. F. Mayers, Numerical Solutions of Partial Differential Equations (Cambridge University Press, 1994).

${ }^{25}$ M. E. Negretti, J.-B. Flor, and E. J. Hopfinger "Development of gravity currents on rapidly changing slopes," J. Fluid Mech. 833, 70-97 (2017).

${ }^{26}$ J. Rottman and J. Simpson, "Gravity currents produced by instantaneous release of a heavy flui in a rectangular channel," J. Fluid Mech. 135, 95-110 (1983).

${ }^{27}$ J. Rottman, J. Simpson, J. Hunt, and R. Britter, "Unsteady gravity current flow over obstacles: Some observations and analysis related to the phase II trials," J. Hazard. Mater. 11, 325-340 (1985).

${ }^{28}$ J. E. Simpson, Gravity Currents in the Environment and the Laboratory (Cambridge University Press, 1997).

${ }^{29} \mathrm{~K}$. Qu, H. S. Tang, and A. Agrawal, "Integration of fully 3D flui dynamics and geophysical flui dynamics models for multiphysics coastal flows Simulation of local complex free-surface phenomena," Ocean Modell. 135, 14-30 (2019).

${ }^{30} \mathrm{M}$. Ungarish, An Introduction to Gravity Currents and Intrusions (Chapman \& Hall/CRC Press, Boca Raton, London, New York, 2009).

${ }^{31}$ M. Ungarish, "Two-layer shallow-water dam-break solutions for nonBoussinesq gravity currents in a wide range of fractional depth," J. Fluid Mech. 675, 27-59 (2011). 